\title{
On the minimal modules for exceptional Lie algebras: Jordan blocks and stabilizers
}

\author{
David I. Stewart
}

\begin{abstract}
Let $G$ be a simple simply connected exceptional algebraic group of type $G_{2}, F_{4}, E_{6}$ or $E_{7}$ over an algebraically closed field $k$ of characteristic $p>0$ with $\mathfrak{g}=\operatorname{Lie}(G)$. For each nilpotent orbit $G \cdot e$ of $\mathfrak{g}$, we list the Jordan blocks of the action of $e$ on the minimal induced module $V_{\min }$ of $\mathfrak{g}$. We also establish when the centralizers $G_{v}$ of vectors $v \in V_{\min }$ and stabilizers $\operatorname{Stab}_{G}\langle v\rangle$ of 1-spaces $\langle v\rangle \subset V_{\text {min }}$ are smooth; that is, when $\operatorname{dim} G_{v}=\operatorname{dim} \mathfrak{g}_{v}$ or $\operatorname{dim} \operatorname{Stab}_{G}\langle v\rangle=\operatorname{dim} \operatorname{Stab}_{\mathfrak{g}}\langle v\rangle$.
\end{abstract}

\section{Introduction}

Let $G$ be a simply connected exceptional algebraic group over an algebraically closed field $k$ of characteristic $p \geqslant 0$ with $\mathfrak{g}=\operatorname{Lie}(G)$. It is a basic fact of the theory of algebraic groups that $G$ is defined over $\mathbb{Z}$; that is, there is a group $G_{\mathbb{Z}}$ such that after extension of scalars to $k$, one gets the group $G$. If $G$ is not of type $E_{8}$, then $G_{\mathbb{Z}}$ admits a non-trivial module $\left(V_{\min }\right)_{\mathbb{Z}}$ of smaller dimension. After reduction modulo $p$, one then gets a module $V_{\min }$ for $G$. If $G=G_{2}, F_{4}, E_{6}$ or $E_{7}$ such a module has dimension $7,26,27$ or 56 , respectively. Recall also that $G$ acts via the adjoint action on its Lie algebra; the associated representation is called the adjoint module. With a classification of unipotent elements in hand, the Jordan block sizes of the action of unipotent elements of $G$ on the adjoint module $\mathfrak{g}$ and minimal module $V_{\text {min }}$ were computed in [9] (see also [10]) and have been extensively used by the mathematical community. Recall that the characteristic $p$ is good for the exceptional group $G$ if $p>3$ and if $G$ is of type $E_{8}$, $p>5$. In good characteristic one has a Springer morphism: a $G$-equivariant bijective map between the variety of unipotent elements of $G$ and the nilpotent cone $\mathcal{N}(\mathfrak{g})$ of $\mathfrak{g}$. Thus the classification of orbits of nilpotent and unipotent elements is the same. At the beginning of $[\mathbf{1 6}$, §3], a reference to a private communication with Lawther indicates that he has checked that the Jordan blocks of unipotent elements and associated nilpotent elements on the adjoint and minimal modules are always the same in good characteristic, with a single exception: on the minimal 56-dimensional module for $E_{7}$ when $p=5$, the regular nilpotent element has blocks of size $23^{2}, 10$ whereas the regular unipotent element has blocks $24,22,10$. A consequence of these calculations is that, together with the remaining Jordan block sizes for nilpotent elements in bad characteristic found in [16], the block sizes on the adjoint module are therefore known in all characteristics. One aim of this note is to compute the Jordan block sizes of nilpotent elements on $V_{\min }$, which are new in bad characteristic. For completeness and ease of use, we have included the block sizes in good characteristic also.

TheOREm 1.1. The Jordan blocks of nilpotent elements $e$ on $V_{\min }$ are listed in Tables 2, 3 and 4 .

As explained above, comparison with the tables in [9] yields the following corollary.

Received 21 September 2015; revised 25 February 2016.

2010 Mathematics Subject Classification 17B45, 20G15 (primary). 
Corollary 1.2 (Lawther). In good characteristic, the Jordan block sizes on $V_{\min }$ of nilpotent and unipotent elements of the same label are the same, unless $p=5$ and $G=E_{7}$, where only the Jordan block sizes of the regular unipotent and nilpotent elements disagree.

Using the calculations in [9], one sees by inspection that the number of Jordan blocks of unipotent elements on the adjoint module is independent of good characteristic; this is reflecting the fact that the centralizers of unipotent elements $G_{u}$ are smooth. Another way of stating this is that the orbit $G \cdot u$ of $u$ is separable, or that $\operatorname{Lie}\left(C_{G}(u)(k)\right)=\mathfrak{c}_{\mathfrak{g}}(u)$. The phenomenon that centralizers are usually smooth holds much more generally; see $[2]$ and, more recently, [5]. It was also noted in [9] when the number of Jordan blocks of a unipotent element on $V_{\min }$ was the same as in characteristic zero. (It turned out that this held in good characteristic.) Thus in good characteristic the scheme of fixed points $\left(V_{\min }\right)^{u}$ is smooth.

We discuss the complementary question in our context, which is possibly more natural. But we must first be a little more precise about $V_{\min }$. Most of the time $V_{\min }$ is irreducible and the theory of high weights identifies $V_{\text {min }}$ uniquely up to isomorphism (possibly after twisting with a graph automorphism in the case of $\left.E_{6}\right)$. The two exceptions are when $(G, p)=\left(F_{4}, 3\right)$ or $\left(G_{2}, 2\right)$. In this case there are essentially two ways in which one may construct a lattice in $\left(V_{\min }\right)_{\mathbb{Z}}$. For one of these, the resulting module after reduction modulo $p$, henceforth $V_{\min }$, has a one-dimensional trivial module in its head with an irreducible $\left(\operatorname{dim} V_{\min }-1\right)$-dimensional socle; this is an induced, co-standard or dual Weyl module for $G$, and $V_{\min }^{*}$ is the corresponding standard or Weyl module for $G$. (Note that for the purposes of computing ranks of powers of matrices, hence Jordan blocks, it matters not whether one works with a module $V$ or its dual.)

With this clarification in hand, let $v \in V_{\min }$ and $\langle v\rangle=k v$ be the 1-space it spans over $k$. Then we establish when the stabilizers $G_{v}$ and $\operatorname{Stab}_{G}\langle v\rangle$ are smooth; by [7, I.7.18(5)] this occurs precisely when $\mathfrak{g}_{v}=\operatorname{Lie}\left(G_{v}(k)\right)$ and $\operatorname{Stab}_{\mathfrak{g}}\langle v\rangle=\operatorname{Lie}\left(\operatorname{Stab}_{G}\langle v\rangle(k)\right)$, respectively. This amounts again to establishing when the orbit $G \cdot v($ or $G \cdot\langle v\rangle)$ is separable.

One consequence is the following theorem.

TheOREm 1.3. The stabilizers $G_{v}$ of vectors $v \in V_{\min }$ and $\operatorname{Stab}_{G}\langle v\rangle$ of 1 -spaces of $V_{\min }$ are smooth whenever $p$ is a good prime for $G$.

We were unable to find any general results in this direction. Thus we ask the following (probably too general) question.

QUESTION 1.4. Let $G$ be a reductive algebraic group over an algebraically closed field $k$ and $V$ be a restricted $G$-module. Under what circumstances are the centralizers $G_{U}$ or stabilizers $\operatorname{Stab}_{G}(U)$ of subschemes $U \subseteq V$ smooth algebraic groups?

Let us reiterate that the paper [5] gives an answer to the question about centralizers when $V$ is the adjoint module for $G$; the correct condition on the characteristic $p$ of $k$ is that it be pretty good for $G$. Also, Cartier's theorem tells us that all algebraic groups are smooth over fields of characteristic zero, so there should be some hope that there are answers which involve a bound on $p$. (Possibly $p>\operatorname{dim} V$ might suffice for the answer to be yes.) Note that $V$ must be restricted for the answer to the question to be interesting: if $V$ is a Frobenius twist $W^{[1]}$ of another non-trivial $G$-module $W$, then $\mathfrak{g}$ acts trivially on $V$, hence centralizes every vector, but $G$ certainly does not. Furthermore, let us underline that already the question is interesting for the case that $U$ consists of a single $k$-point of $V$. The part of the question dealing with stabilizers is much less likely to have a nice answer: see [6] which basically gives the answer for the corresponding question about normalizers of subspaces on the adjoint module. 


\subsection{Comparison with UGA VIGRE results}

During the process of making these computations, it was discovered that there are a number of errors in the tables of representatives of nilpotent orbits in [16]. In the appendix we have provided a complete list of corrected representatives derived from $[\mathbf{1 3}]$ and $[\mathbf{1 4}]$ that can be used to fix the UGA VIGRE tables.

The first set of (minor) errors involve the transcription of representatives from the MAGMA code used into $\mathrm{LT}_{\mathrm{E} X} \mathrm{X}$, yet the stated Jordan block sizes remain correct. These orbit representatives are:

- $F_{4}\left(a_{3}\right)$ in $F_{4}$;

- $A_{6}^{(2)}$ in $E_{8}$;

- $E_{7}\left(a_{1}\right)$ in $E_{8}$;

- $E_{8}\left(a_{2}\right), E_{8}\left(a_{3}\right), E_{8}\left(a_{4}\right)$ in $E_{8}$.

With these corrections the UGA VIGRE representatives are correct for $p=0$ and $p \geqslant 5$.

The second set of errors orbits involve the following orbits in characteristics 2 or 3 :

- $D_{r}\left(a_{1}\right), D_{r}\left(a_{1}\right)+A_{s}, p=2$

- $D_{r}\left(a_{2}\right), D_{r}\left(a_{2}\right)+A_{s}, p=3$

- $E_{8}\left(b_{6}\right), p=2$ and 3 .

The problem is that the aforementioned listed representatives in [16] break down in either characteristic 2 or 3 , meaning that the Jordan block sizes are not correct in these cases. One can use the tables in the appendix for these orbits and their Jordan blocks to correct the tables in [16]. In fact, we give all adjoint Jordan block sizes in all cases for completeness, even where they are known to coincide with those in $[\mathbf{9}]$ for good characteristic.

\section{Jordan blocks}

We describe the method of computation of the tables of Jordan blocks. All this was done in GAP. For convenience, we locate the relevant modules $V_{\min }$ in subquotients of nilradicals of parabolic subalgebras of $\mathfrak{g}$. The general theory on the structure of nilradicals of parabolic subalgebras of reductive Lie algebras $\mathfrak{g}=\operatorname{Lie}(G)$ (and the group-theoretic analogues for $G$ ) can be found in $[\mathbf{1}]$. With the notation of $[\mathbf{3}]$ we will give a description in terms of roots. In $\mathfrak{g}$ of type $E_{8}$ with Cartan subalgebra $\mathfrak{h}$, we locate a Levi subalgebra $\mathfrak{l}$ of type $E_{7}$ containing $\mathfrak{h}$ and corresponding roots of the form

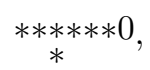

where each value of $*$ is taken arbitrarily so that the result is a root. Then the derived subalgebra $\mathfrak{l}^{\prime}$ is simple of type $E_{7}$ and acts by derivations on the space spanned by the 56 positive roots of the form

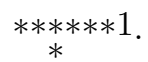

An analysis of the highest weight shows that this is indeed the 56 -dimensional module $V_{\min }=$ $V_{56}$. Similarly, one locates $E_{6}$ in $E_{7}$ corresponding to roots $* * * * * 0$ acting on a 27-dimensional minimal module $V_{\min }=V_{27}$ corresponding to roots $* * * * * 1$. Nilpotent orbit representatives for $E_{6}$ and $E_{7}$ in all characteristics in terms of a sum of simple root spaces are available from [13] and $[\mathbf{1 4}]$.

Now one realizes all these elements in GAP. The package LieAlgebras in the standard distribution of GAP4 will construct a simple Lie algebra $\mathfrak{g}$ of rank $r$ and number of positive roots $n=\left|R^{+}\right|$over the rationals which comes with a 'canonical' Chevalley basis $B$. This 
has $\{B[1], \ldots, B[r]\}$ as a basis for the simple root spaces, $\{B[1], \ldots, B[n]\}$ as a basis for the positive root spaces; for $1 \leqslant i \leqslant n$ we have that $B[n+i]$ spans the root space corresponding to the negative root to which $B[i]$ corresponds, and $\{B[2 n+1], \ldots, B[2 n+r]\}$ spans a Cartan subalgebra. Further, if $a, b \in \mathfrak{g}$, then the operation $\mathrm{a} * \mathrm{~b}$ returns the commutator $[a, b]$ expressed via the $B[i]$. It is a simple matter to write any nilpotent representative $e$ as a sum of a subset of the $B[i]$ and also identify those $B[i]$ which span a basis $B_{V}$ of $V_{\min }$ as described in the previous paragraph. One may then ask GAP to compute the action of $e$ on $V_{\min }$ as a matrix over the basis $B_{V}$ by hitting each vector of $B_{V}$ with $e$ and re-expressing this as a linear combination of elements of $B_{V}$. This associates $e$ to a $\left(\operatorname{dim} V_{\min } \times \operatorname{dim} V_{\min }\right)$ matrix $M_{e}$ whose entries are integers by virtue of the fact that $B$ was a Chevalley basis.

The Jordan blocks of $e$ are then determined by the ranks of successive powers of $M_{e}$. We first run a routine which bounds the number of exceptional primes whose Jordan block structure differs from the generic Jordan block structure as seen over $\mathbb{Q}$. This works simply by taking the union over all primes dividing the elementary divisors of each power of $M_{e}$. Then the Jordan block structure is output over $\mathbb{Q}$, together with the Jordan block structure over $\mathbb{F}_{p}$ for any exceptional $p$. The result for $E_{6}$ and $E_{7}$ is then output in the tables below.

The situation for the seven- and 26-dimensional minimal modules for the Lie algebras of type $G_{2}$ and $F_{4}$ is treated similarly, but one must work just a little harder. One has that $F_{4}$ is a subalgebra of $E_{6}$, such that the $E_{6}$-module $V_{27}$ has restriction to $F_{4}$ which is $V_{\min } \oplus k$ unless $p=3$ and $V_{27} \mid F_{4}$ is uniserial with successive factors $k$, $\operatorname{soc} V_{\min }$, and $k$. (It is in fact a tilting module.) If $p \neq 3$ then the module $V_{\min }$ is irreducible and self-dual, so that for all $p$ there is a quotient isomorphic to $V_{\min }$ of dimension 26 . Now $F_{4}$ is located in $E_{6}$ by sending the simple root vector $e_{\alpha_{1}}$ to $e_{\alpha_{2}}^{\prime}, e_{\alpha_{2}}$ to $e_{\alpha_{4}}^{\prime}, e_{\alpha_{3}}$ to $e_{\alpha_{3}}^{\prime}+e_{\alpha_{5}}^{\prime}$ and $e_{\alpha_{4}}$ to $e_{\alpha_{1}}^{\prime}+e_{\alpha_{6}}^{\prime}$. The remaining positive root elements of $F_{4}$ expressed in terms of those of $E_{6}$ can be generated from this. If $V_{27}$ has basis $B_{V}$ it is straightforward to locate a vector stabilized by $F_{4}$ and then form the matrix of the action of each element $e$ on the quotient; this gives a $26 \times 26$ matrix $M_{e}$. One then repeats the procedure as described above for computing the Jordan blocks.

Finally, a similar procedure locates $G_{2}$ in a $D_{4}$ Levi subalgebra of $E_{6}$ sending $e_{\alpha_{1}}$ to $e_{\alpha_{2}}^{\prime}+$ $e_{\alpha_{3}}^{\prime}+e_{\alpha_{5}}^{\prime}$ in $E_{6}$ and $e_{\alpha_{2}}$ to $e_{\alpha_{4}}^{\prime}$ in $E_{6}$. Then the eight-dimensional natural module $V_{8}$ for $D_{4}$ corresponding to roots $0 * * * 0$ is obtained via the roots $1 * * * 0$. The restriction of $V_{8}$ to the $G_{2}$-subalgebra contains a trivial submodule such that the quotient is isomorphic to $V_{\min }$ as before.

\section{Smoothness of centralizers and stabilizers}

In this section we consider simple, simply connected algebraic groups of type $G:=G_{2}, F_{4}$, $E_{6}$ and $E_{7}$ over algebraically closed fields $k$ of arbitrary characteristic acting on their minimal modules $V_{\min }:=V_{7}, V_{26}, V_{27}$ and $V_{56}$, of dimensions 7, 26, 27 and 56, respectively. The stabilizers and centralizers of 1-spaces of $V_{\min }$ for the corresponding finite groups $G_{2}\left(\mathbb{F}_{q}\right)$, $F_{4}\left(\mathbb{F}_{q}\right), E_{6}\left(\mathbb{F}_{q}\right)$ and $E_{7}\left(\mathbb{F}_{q}\right)$ are well known to group theorists and we record the extension which gives the reduced part $\left(\operatorname{Stab}_{G}\langle v\rangle\right)_{\text {red }}$ of the scheme-theoretic stabilizers $\operatorname{Stab}_{G}\langle v\rangle$ in $G$ of the 1space $\langle v\rangle \in V_{\min }$ in the next lemma. (Recall that for an algebraic group $K$, over an algebraically closed field $k$, not necessarily smooth, one may associate a smooth algebraic group $K_{\text {red }} \subseteq K$ such that $K_{\text {red }}(k)=K(k)$.) We prove, using computational methods, that $\operatorname{Stab}_{G}\langle v\rangle$ and $G_{v}$ are smooth provided $p$ is a good prime, so that for these primes, $\left(\operatorname{Stab}_{G}\langle v\rangle\right)_{\text {red }}=\operatorname{Stab}_{G}\langle v\rangle$ and the Lie-theoretic stabilizer is recovered as $\operatorname{Lie}\left(\left(\operatorname{Stab}_{G}\langle v\rangle\right)_{\text {red }}\right)$.

LEmma 3.1. (i) If $G$ is of type $E_{7}$ then $G(k)$ has four orbits on the 1-spaces of $V:=V_{56}$. If $0 \neq\langle v\rangle \subseteq V$ then the stabilizer $\left(\operatorname{Stab}_{G}\langle v\rangle\right)_{\text {red }}$ is a closed subgroup of $G$ isomorphic to one of: 
(a) an $E_{6}$ parabolic subgroup of $G$;

(b) the semidirect product of an $E_{6}$ Levi subgroup of $G$ with an involution inducing a graph automorphism on $E_{6}$;

(c) a subgroup of a $D_{6}$-parabolic subgroup $P$ of $G$ isomorphic to $B_{5} T_{1} R_{u}(P)$;

(d) a subgroup of an $E_{6}$-parabolic $P$ of $G$ equal to the semidirect product $H R_{u}(P)$, where $H$ is a subgroup of the Levi subgroup $L$ of $P$ of type $F_{4} T_{1}$.

(ii) If $G$ is of type $E_{6}$ then $G(k)$ has three orbits on the 1-spaces of $V:=V_{27}$. If $0 \neq\langle v\rangle \subseteq V$ then the stabilizer $\left(\operatorname{Stab}_{G}\langle v\rangle\right)_{\text {red }}$ is a closed subgroup of $G$ isomorphic to one of:

(a) a $D_{5}$-parabolic subgroup $P$ of $G$;

(b) a subgroup of a $D_{5}$-parabolic subgroup $P$ equal to the semidirect product $H R_{u}(P)$, where $H$ is a subgroup of the Levi subgroup $L$ of $P$ of type $B_{4} T_{1}$;

(c) a subgroup of $G$ of type $F_{4}$.

(iii) If $G$ is of type $F_{4}$ then $G(k)$ has infinitely many orbits on the 1-spaces of $V:=V_{26}$. If $0 \neq\langle v\rangle \subseteq V$ and $p \neq 3$ then the stabilizer $\left(\operatorname{Stab}_{G}\langle v\rangle\right)_{\text {red }}$ is isomorphic to one of:

(a) $B_{4}$ (one orbit);

(b) a $B_{3}$-parabolic subgroup $P$ of $G$ (one orbit);

(c) a subgroup of $P$ isomorphic to $G_{2} T_{1} \ltimes k^{14}$ (one orbit);

(d) a subgroup of $P$ isomorphic to $B_{3} \ltimes k^{7}$ (one orbit);

(e) a 28-dimensional subgroup such that the connected component $\left(\left(\operatorname{Stab}_{G}\langle v\rangle\right)_{\mathrm{red}}\right)^{\circ}$ has type $D_{4}$. In this case, each orbit contains a unique element in one of the $k \backslash\{0\}$ generic 1-spaces of the 0 -weight space of $F_{4}$ on $V_{26}$.

(iv) If $G$ is of type $G_{2}$ then $G(k)$ has two orbits on the set of 1-spaces of $V:=V_{7}$. If $0 \neq\langle v\rangle \subseteq V$ then the stabilizer $\left(\operatorname{Stab}_{G}\langle v\rangle\right)_{\text {red }}$ is isomorphic to one of:

(a) a long $A_{1}$-parabolic subgroup of $G$;

(b) $A_{2} .2$ for a subsystem subgroup of type $A_{2}$ consisting of long roots.

Proof. Let $H:=\left(\operatorname{Stab}_{G}\langle v\rangle\right)_{\text {red. }}$ First of all, observe that $H$, as a subgroup of the $\mathbb{Z}$-defined embedding of $G$ into $\mathrm{GL}(V)$ for $V=V_{27}$ or $V_{56}$, is defined over $\mathbb{Z}$, hence certainly over $\overline{\mathbb{F}}_{p}$. We have $H_{\overline{\mathbb{F}}_{p}}\left(\overline{\mathbb{F}}_{p}\right)=\bigcup_{r \geqslant 0} H_{\overline{\mathbb{F}}_{p}}\left(\mathbb{F}_{p^{r}}\right)$, for instance by intersecting with $\operatorname{GL}(V)\left(\mathbb{F}_{p^{r}}\right)$. Since $H$ is smooth, the $\overline{\mathbb{F}}_{p}$-points of $H$ are dense in $H$ and so we have that the union $\bigcup_{r \geqslant 0} H_{\overline{\mathbb{F}}_{p}}\left(\mathbb{F}_{p^{r}}\right)$ is dense in $H$.

Assume we are not in case (iii)(e) or (iv) in characteristic 2. Then the structure as stated, in view of [15, I.2.7], follows directly from [4, p. 467 and Table 2] (for $E_{6}$ and $F_{4}$ ), [12, Lemma 4.3] (for $E_{7}$ ) and [8, Proposition 2.2] (for $G_{2}$ ). In reading those references, note that twisted subgroups such as ${ }^{2} A_{2}(q)$ occur in the presence of a stabilizer $\operatorname{Stab}_{G}\langle v\rangle$ of the form $H \cdot\langle\tau\rangle$ where $\tau$ is a graph automorphism of $H$. Then the orbit $G \cdot v$ splits into $\left|\mathrm{H}^{1}\left(F, G_{v} / G_{v}^{\circ}\right)\right|$ orbits under $G\left(\mathbb{F}_{p^{r}}\right)$, by $[\mathbf{1 5}$, I.2.7].

A little more work is necessary to understand (iii)(e). It is shown in [4] that each 1-space not conjugate to any previously considered is conjugate to a subspace of a 'special plane' $\pi=\left\langle e_{1}, e_{2}, e_{3}\right\rangle$ (in [4] a 'plane' is a plane of $\mathbb{P}\left(V_{27}\right)$, hence a 3-space of $V_{27}$ ). Moreover, all such special planes are conjugate under the action of $E_{6}$ and one may choose the $e_{i}$ to be weight vectors for $E_{6}$. Since $F_{4}$ is in fact the stabilizer of an element $e=e_{1}+e_{2}+e_{3}$ of $\pi$, it is not hard to check that the 0 -weight space for a maximal torus $F_{4}$ in $V_{27} /\langle e\rangle$ is $\pi /\langle e\rangle$. Then a generic 1-space in $\pi /\langle e\rangle$ is $\left\langle e_{1}+t \cdot e_{2}\right\rangle+\langle e\rangle$ for $t \neq 0,1$. In light of [4], the stabilizers in $G$ of all generic 1-spaces are then seen to satisfy the conditions in (iii)(e) as stated.

For (iv) in characteristic 2, note that the stabilizers of the 1-spaces given are both maximal smooth subgroups $H_{1}$ and $H_{2}$ of $G$ which are $\mathbb{Z}$-defined. After reduction modulo 2, we will therefore have containments $H_{1} \subseteq\left(\operatorname{Stab}_{G}\left\langle v_{1}\right\rangle\right)_{\text {red }}$ and $H_{2} \subseteq\left(\operatorname{Stab}_{G}\left\langle v_{2}\right\rangle\right)_{\text {red. }}$. It cannot be the case that either $\operatorname{Stab}_{G}\left\langle v_{1}\right\rangle$ or $\operatorname{Stab}_{G}\left\langle v_{2}\right\rangle$ is the whole of $G$, since $G$ has no fixed 1-spaces on $V_{\text {min }}$, so the isomorphism types of $\left(\operatorname{Stab}_{G}\langle v\rangle\right)_{\text {red }}$ must be as given. To see that the number of 
orbits is still the same, one counts the number of elements in orbits of 1-spaces for $G_{2}(q)$. If $P$ is a long root parabolic of $G_{2}(q)$ it is an easy check that

$$
\left|G_{2}(q)\right| \cdot\left(\frac{1}{|P(q)|}+\frac{1}{\left|A_{2}(q) \cdot 2\right|}+\frac{1}{\left|{ }^{2} A_{2}(q) \cdot 2\right|}\right)=\frac{q^{7}-1}{q-1}
$$

as required.

Using GAP, we find that the group-theoretic and infinitesimal stabilizers of 1-spaces correspond.

TheOREM 3.2. Let $G$ be simple and simply connected of type $E_{7}$ (respectively, $E_{6}, F_{4}, G_{2}$ ) and let $V=V_{56}$ (respectively, $V=V_{27}, V_{26}, V_{7}$ ) be a minimal-dimensional non-trivial induced module for $G$.

Then the stabilizers in $G$ of vectors and 1-spaces of $V$ are smooth (that is, $\operatorname{dim} G_{v}=\operatorname{dim} \mathfrak{g}_{v}$ and $\left.\operatorname{dim} \operatorname{Stab}_{G}\langle v\rangle=\operatorname{dim} \operatorname{Stab}_{\mathfrak{g}}\langle v\rangle\right)$, with the following exceptions.

(i) If $(G, p)=\left(E_{7}, 2\right)$, then $G_{v}$ and $\operatorname{Stab}_{G}\langle v\rangle$ are not smooth if $\langle v\rangle$ has stabilizer of type $F_{4} T_{1} \ltimes k^{26}$.

(ii) If $(G, p)=\left(E_{7}, 2\right)$, then $\operatorname{Stab}_{G}\langle v\rangle$ is not smooth if $\langle v\rangle$ has stabilizer of type $E_{6} .2$.

(iii) If $(G, p)=\left(E_{6}, 3\right)$, then $\operatorname{Stab}_{G}\langle v\rangle$ is not smooth if $\langle v\rangle$ has stabilizer of type $F_{4}$.

(iv) If $(G, p)=\left(F_{4}, 2\right)$, then $\operatorname{Stab}_{G}\langle v\rangle$ is not smooth if $\langle v\rangle$ has stabilizer of type $B_{3} \ltimes k^{7}$.

(v) If $(G, p)=\left(F_{4}, 3\right)$, then $G_{v}$ and $\operatorname{Stab}_{G}\langle v\rangle$ are not smooth if $\langle v\rangle$ has stabilizer of type $G_{2} T_{1} \ltimes k^{14}$.

(vi) If $(G, p)=\left(G_{2}, 2\right)$, then $G_{v}$ and $\operatorname{Stab}_{G}\langle v\rangle$ are not smooth if $\langle v\rangle$ has stabilizer which is a long $A_{1}$-parabolic.

Proof. Let $v \in V=V_{\text {min }}$ and set $K:=\left(\operatorname{Stab}_{G}\langle v\rangle\right)_{\text {red }}$. Then certainly we have a containment $\operatorname{Lie}(K)=\operatorname{Lie}\left(\operatorname{Stab}_{G}\langle v\rangle\right)_{\mathrm{red}} \subseteq \operatorname{Stab}_{\mathfrak{g}}\langle v\rangle=: \mathfrak{k}$. We wish to show that equality holds. For this, it suffices to show that $\operatorname{dim} \operatorname{Stab}_{\mathfrak{g}}\langle v\rangle=\operatorname{dim}\left(\operatorname{Stab}_{G}\langle v\rangle\right)_{\text {red. }}$. The values of the right-hand side are provided by Lemma 3.1 .

To prove the equality of dimensions, we work with GAP in the following way.

(i) Construct $V_{\min }$ in GAP as in the previous section with $\mathfrak{g}$ contained in a Levi subalgebra $\mathfrak{l}$ of a parabolic $\mathfrak{p}=\mathfrak{l}+\mathfrak{q} \subseteq \mathfrak{h}$ for $\mathfrak{h}=E_{6}, E_{7}$ or $E_{8}$, such that $V_{\text {min }}$ is contained as a quotient of the unipotent radical $\mathfrak{q}$ of $\mathfrak{p}$.

(ii) Search for representatives for the $G$-orbits on $V$. Since one knows that the finite number of orbits in case $G$ is of type $E_{6}, E_{7}$ and $G_{2}$, one simply seeks this number of nonisomorphic stabilizers in $G$. (Guidance was provided in [4, p. 467] and [12, Lemma 4.3].) For $F_{4}$, except for stabilizers of type $D_{4}$, a similar procedure works, and the stabilizers of type $D_{4}$ are described explicitly in Lemma 3.1. Representatives are given in Table 1.

Assume for the moment that $G$ is not of type $F_{4}$ or $\langle v\rangle$ does not have stabilizer of type $D_{4}$.

(iii) For each element $b$ in a Chevalley basis $B_{1}$ of $\mathfrak{g}$, calculate the coefficients of $[b, v]$ reexpressed in terms of the Chevalley basis $B$ of $\mathfrak{h}$.

(iv) Form the matrix $M$ of these coefficients (which is integral, by our choice of representatives in Table 1) and calculate its elementary divisors. It turns out that unless we are in one of the exceptional cases, all elementary divisors are either 1 or 0 , hence the rank $r$ of this matrix will not change after reduction modulo $p$.

(v) We have $\operatorname{dim} \mathfrak{g}-r=\operatorname{dim} \mathfrak{g}_{v}$. If $v \in \mathfrak{g} \cdot v$ then $\operatorname{dim} \mathfrak{g}_{v}=\operatorname{dim} \operatorname{Stab}_{\mathfrak{g}}\langle v\rangle-1$. Otherwise, $\operatorname{dim} \mathfrak{g}_{v}=\operatorname{dim} \operatorname{Stab}_{\mathfrak{g}}\langle v\rangle$. To establish which, we simply add a new line to the matrix $M$ containing the coefficients of $v$ in terms of $B$ and take its elementary divisors again.

(vi) It turns out that, apart from the exceptional cases, $\operatorname{dim} \mathfrak{g}_{v}=\operatorname{dim} G_{v}$ and $\operatorname{dim} \operatorname{Stab}_{\mathfrak{g}}\langle v\rangle=$ $\operatorname{dim} \operatorname{Stab}_{G}\langle v\rangle$. 
To deal with the case where $G=F_{4}$ and $\langle v\rangle$ has stabilizer of type $D_{4}$, we perform a similar calculation with $\mathfrak{g}[t]$ and working with matrices over $\mathbb{Z}[t]$. Let $v=e_{1}+t e_{2}$ be a generic element in the 0 -weight space of $V_{\min }$. Form $M$ as before to get a $26 \times 52$ matrix. It turns out that 28 of these rows are identically zero and so the rank will not change after they are removed. It also turns out that for $t \neq 0,1$, the resulting $26 \times 24$ matrix has, for any choice of $t$, a single non-zero entry in each row, with no two non-zero entries in a common column. Thus the rank of the matrix is 24 in all characteristics for all choices of $t \neq 0,1$, and we are done.

Remarks 3.3. Except when $(G, p)=\left(G_{2}, 2\right)$, for each exceptional case from Lemma 3.2 we have that the group-theoretic stabilizer is one fewer dimension than that in the Lie algebra. In each case, there is an extra toral element which stabilizes $v$ or $\langle v\rangle$ which is not in the Lie algebra of the reduced part of the group-theoretic stabilizer.

For $(G, p)=\left(G_{2}, 2\right)$, the non-smoothness of the stabilizer of the 1-space whose reduced part is an $A_{1}$-parabolic (which is a maximal smooth subgroup) implies that there is a maximal rank subalgebra $\mathfrak{h}=\mathfrak{g}_{v}$ containing an $A_{1}$-parabolic subalgebra of $\mathfrak{g}$ which is not the Lie algebra of any smooth subgroup of $G$, hence is not obtained using the Borel-de Siebenthal algorithm. For our representative $v$,

$$
\operatorname{Stab}_{\mathfrak{g}}\langle v\rangle=\left\langle e_{\alpha_{2}}, e_{\alpha_{4}}, e_{-\alpha_{1}}, e_{-\alpha_{2}}, e_{-\alpha_{3}}, e_{-\alpha_{4}}, e_{-\alpha_{5}}, e_{-\alpha_{6}}, h_{1}, h_{2}\right\rangle
$$

where $\left\langle h_{1}, h_{2}\right\rangle$ is a Cartan subalgebra of $\mathfrak{g}$. This is a long $A_{1}$-parabolic after throwing in the outstanding root subspace $\left\langle e_{\alpha_{4}}\right\rangle$ of the short $\tilde{A}_{1}$ subalgebra which commutes with the $A_{1}$ Levi $\left\langle e_{ \pm \alpha_{2}}, h_{1}, h_{2}\right\rangle$.

For $G_{2}$ in characteristic 2, there are many more such maximal rank subalgebras, including one of dimension 11. These are discussed in some generality in [11]. One reason for the explosion in possibilities is the fact that in characteristic 2, one has, remarkably, an isomorphism of Lie algebras $\mathfrak{g} \cong \mathfrak{p s l}_{4}$.

Finally, let us remark also that when $p=2$, the Lie algebras $G_{2} T_{1} \ltimes k^{14}$ and $B_{3} T_{1} \ltimes k^{7}$ are isomorphic; thus, while the stabilizers of the 1-spaces in the relevant orbits in $V_{26}$ are not isomorphic algebraic groups, their Lie algebra stabilizers are.

TABLE 1. Representatives of the orbits of $E_{6}$ and $E_{7}$ on minimal modules.

\begin{tabular}{llll}
\hline$G$ & $\left(\operatorname{Stab}_{G}\langle v\rangle\right)_{\text {red }}$ & Representative in $\mathfrak{q}$ & $\operatorname{dim} \operatorname{Stab}_{g}\langle v\rangle-\operatorname{dim} \mathfrak{g}_{v}$ \\
\hline$G_{2}$ & $A_{2}$ & $e_{\alpha_{1}+\alpha_{3}+\alpha_{4}}$ & 0 \\
& $A_{1}$-parabolic & $e_{\alpha_{1}}$ & 1 \\
\hline$F_{4}$ & $B_{4}$ & $e_{112211}$ & 0 \\
& $B_{3} T_{1} \ltimes k^{14}$ & $e_{\alpha_{7}}$ & 1 \\
& $G_{2} T_{1} \ltimes k^{14}$ & $e_{\alpha_{7}}+e_{134232}$ & 1 \\
& $B_{3} \ltimes k^{7}$ & $e_{\alpha_{7}}+e_{122111}+e_{134321}$ & $0(1$ if $p=2)$ \\
& $D_{4}$ & $e_{122111}+t \cdot e_{112211}, t \neq 0,1$ & 0 \\
\hline$E_{6}$ & $D_{5}$-parabolic & $e_{\alpha_{7}}$ & 1 \\
& $B_{4} T_{1} \ltimes k^{16}$ & $e_{\alpha_{7}}+e_{\tilde{\alpha}}$ & 1 \\
& $F_{4}$ & $e_{122111}+e_{112211}^{1}+e_{012221}$ & $0(1$ if $p=3)$ \\
\hline$E_{7}$ & $E_{6}$-parabolic & $e_{\alpha_{8}}$ & 1 \\
& $F_{4} T_{1} \ltimes k^{26}$ & $e_{2343221}+e_{1343321}+e_{1244321}$ & 1 \\
& $B_{5} T_{1} \ltimes k^{1+32}$ & $e_{2354321}+e_{2454321}$ & 1 \\
& $E_{6} .2$ & $e_{\alpha_{8}}+e_{\tilde{\alpha}-\alpha_{8}}$ & $0(1$ if $p=2)$ \\
\hline
\end{tabular}


TABLE 2. Jordan blocks of nilpotent elements on the module $V_{56}$ for $E_{7}, I$.

\begin{tabular}{|c|c|c|c|c|c|}
\hline$e$ & $p$ & Jordan blocks & $e$ & $p$ & Jordan blocks \\
\hline \multirow[t]{6}{*}{$E_{7}$} & 2 & $14^{4}$ & \multirow[t]{5}{*}{$\overline{E_{7}\left(a_{5}\right)}$} & 2 & \multirow{5}{*}{$\begin{array}{l}8^{4}, 6^{2}, 4^{2}, 2^{2} \\
9^{2}, 8,6^{3}, 4^{3} \\
10,8^{2}, 6,5^{4}, 4 \\
7^{6}, 6,4^{2} \\
10,8^{2}, 6^{3}, 4^{3}\end{array}$} \\
\hline & 3 & $19^{2}, 18$ & & 3 & \\
\hline & 5 & $23^{2}, 10$ & & 5 & \\
\hline & 7 & $28,14^{2}$ & & 7 & \\
\hline & 19 & $19^{2}, 18$ & & others & \\
\hline & $\begin{array}{l}23 \\
\text { others }\end{array}$ & $\begin{array}{l}23^{2}, 10 \\
28,18,10\end{array}$ & \multirow[t]{2}{*}{$D_{5}$} & $\begin{array}{l}2 \\
3\end{array}$ & $\begin{array}{l}8^{4}, 5^{4}, 1^{4} \\
9^{2}, 8^{4}, 1^{6}\end{array}$ \\
\hline \multirow[t]{6}{*}{$E_{7}\left(a_{1}\right)$} & \multirow{6}{*}{$\begin{array}{l}2 \\
3 \\
7 \\
11 \\
17 \\
19 \\
\text { others }\end{array}$} & \multirow{6}{*}{$\begin{array}{l}14^{4} \\
19^{2}, 9^{2} \\
22,14^{2}, 6 \\
22,12,11^{2} \\
17^{2}, 16,6 \\
19^{2}, 12,6 \\
22,16,12,6 \\
\end{array}$} & & others & $11^{2}, 9^{2}, 5^{2}, 1^{6}$ \\
\hline & & & \multirow[t]{4}{*}{$E_{6}\left(a_{3}\right)$} & 2 & $8^{4}, 5^{4}, 1^{4}$ \\
\hline & & & & 3 & $9^{2}, 6^{4}, 3^{4}, 1^{2}$ \\
\hline & & & & & $7^{6}, 5^{2}, 1^{4}$ \\
\hline & & & & others & $9^{2}, 7^{2}, 5^{4}, 1^{4}$ \\
\hline & & & \multirow[t]{4}{*}{$\overline{D_{6}\left(a_{2}\right)}$} & & $8^{2}, 6^{6}, 2^{2}$ \\
\hline \multirow{4}{*}{$E_{7}\left(a_{2}\right)$} & \multirow{4}{*}{$\begin{array}{l}2 \\
3 \\
13 \\
17 \\
\text { others } \\
\end{array}$} & \multirow{4}{*}{$\begin{array}{l}14^{2}, 12^{2}, 2^{2} \\
18,10^{3}, 8 \\
13^{4}, 4 \\
17^{2}, 10,8,4 \\
18,16,10,8,4 \\
\end{array}$} & & 5 & $10,8,7^{2}, 5^{4}, 4$ \\
\hline & & & & & $7^{6}, 5^{2}, 4$ \\
\hline & & & & others & $10,8,7^{2}, 6,5^{2}, 4^{2}$ \\
\hline & & & \multirow[t]{3}{*}{$D_{5}\left(a_{1}\right)+A_{1}$} & & $8^{2}, 6^{2}, 4^{6}, 2^{2}$ \\
\hline \multirow[t]{3}{*}{$E_{7}\left(a_{3}\right)$} & \multirow{3}{*}{$\begin{array}{l}2 \\
11 \\
13 \\
\text { others }\end{array}$} & \multirow{3}{*}{$\begin{array}{l}14^{2}, 8^{2}, 6^{2} \\
11^{4}, 10,2 \\
13^{2}, 12,10,6,2 \\
16,12,10^{2}, 6,2\end{array}$} & & & $7^{6}, 4,2^{5}$ \\
\hline & & & & others & $8^{3}, 6^{3}, 4,2^{5}$ \\
\hline & & & \multirow[t]{3}{*}{$A_{5}+A_{1}$} & & $8^{2}, 6^{6}, 2^{2}$ \\
\hline \multirow[t]{3}{*}{$E_{6}$} & \multirow{3}{*}{$\begin{array}{l}2 \\
3 \\
13 \\
\text { others }\end{array}$} & \multirow{3}{*}{$\begin{array}{l}13^{4}, 1^{4} \\
9^{6}, 1^{2} \\
13^{4}, 1^{4} \\
17^{2}, 9^{2}, 1^{4}\end{array}$} & & & $7^{4}, 6^{3}, 5^{2}$ \\
\hline & & & & others & $10,7^{2}, 6^{3}, 5^{2}, 4$ \\
\hline & & & $\left(A_{5}\right)^{\prime}$ & 2 & $7^{4}, 6^{4}, 1^{4}$ \\
\hline \multirow[t]{3}{*}{$E_{6}\left(a_{1}\right)$} & \multirow{3}{*}{$\begin{array}{l}3 \\
7 \\
11 \\
\text { others }\end{array}$} & & & & $\begin{array}{l}9^{2}, 6^{4}, 3^{4}, 1^{2} \\
7^{4}, 6^{4}, 1^{4}\end{array}$ \\
\hline & & $13^{2}, 7^{4}, 1^{2}$ & & others & $9^{2}, 6^{4}, 5^{2}, 1^{4}$ \\
\hline & & $\begin{array}{l}11^{4}, 5^{2}, 1^{2} \\
13^{2}, 9^{2}, 5^{2}, 1^{2}\end{array}$ & $A_{4}+A_{2}$ & & $7^{4}, 4^{4}, 3^{4}$ \\
\hline$D_{6}$ & 2 & $8^{4}, 6^{4}$ & & & $5^{10}, 3^{2}$ \\
\hline & & $11^{4}, 10,1^{2}$ & & others & $7^{4}, 5^{2}, 3^{6}$ \\
\hline & $\begin{array}{l}13 \\
\text { others }\end{array}$ & $\begin{array}{l}13^{2}, 11^{2}, 6,1^{2} \\
16,11^{2}, 10,6,1^{2}\end{array}$ & $D_{5}\left(a_{1}\right)$ & & $8^{2}, 5^{4}, 4^{4}, 1^{4}$ \\
\hline$E_{7}\left(a_{4}\right)$ & 2 & $8^{4}, 6^{4}$ & & others & $8^{2}, 7^{2}, 6^{2}, 3^{2}, 2^{2}, 1^{4}$ \\
\hline & & $\begin{array}{l}9^{4}, 7^{2}, 3^{2} \\
12,10^{2}, 5^{2}, 4.2\end{array}$ & $A_{4}+A_{1}$ & & $5^{10}, 2^{2}, 1^{2}$ \\
\hline & & $\begin{array}{l}12,10^{2}, 8,5^{2}, 4,2 \\
11^{2} 10864^{2} 2\end{array}$ & & others & $7^{2}, 6^{2}, 5^{2}, 4^{2}, 3^{2}, 2^{2}, 1^{2}$ \\
\hline & others & $12,10^{2}, 8,6,4^{2}, 2$ & $D_{4}+A_{1}$ & 2 & $4^{12}, 2^{4}$ \\
\hline$D_{6}\left(a_{1}\right)$ & $\begin{array}{l}2 \\
3\end{array}$ & $\begin{array}{l}8^{4}, 6^{4} \\
9^{4}, 7^{2}, 3^{2}\end{array}$ & & $\begin{array}{l}7 \\
\text { others }\end{array}$ & $\begin{array}{l}7^{6}, 2^{5}, 1^{4} \\
8,7^{4}, 6,2^{5}, 1^{4}\end{array}$ \\
\hline & & $12,10,9^{2}, 5^{2}, 3^{2}$ & $\left(A_{5}\right)^{\prime \prime}$ & 2 & $8^{2}, 6^{6}, 2^{2}$ \\
\hline & & $11^{2}, 9^{2}, 6,4,3^{2}$ & & & $7^{2}, 6^{7}$ \\
\hline & others & $12,10,9^{2}, 6,4,3^{2}$ & & & $7^{2}, 6^{7}$ \\
\hline$D_{5}+A_{1}$ & 2 & $8^{4}, 6^{2}, 4^{2}, 2^{2}$ & & others & $10,6^{7}, 4$ \\
\hline & & $9^{2}, 8^{4}, 2^{3}$ & $A_{3}+A_{2}+A_{1}$ & 2 & $4^{12}, 2^{4}$ \\
\hline & others & $11^{2}, 10,8,5^{2}, 2^{3}$ & & & $6^{3}, 4^{3}, 3^{8}, 2$ \\
\hline$\left(A_{6}\right)^{(2)}$ & 2 & $8^{4}, 6^{2}, 4^{2}, 2^{2}$ & & $\begin{array}{l}5 \\
\text { others }\end{array}$ & $\begin{array}{l}5^{6}, 4^{4}, 2^{5} \\
6^{3}, 4^{7}, 2^{5}\end{array}$ \\
\hline$A_{6}$ & 2 & & $A_{4}$ & & $5^{10}, 1^{6}$ \\
\hline & & $\begin{array}{l}9^{4}, 7^{2}, 3^{2} \\
7^{8}\end{array}$ & & others & $7^{2}, 5^{6}, 3^{2}, 1^{6}$ \\
\hline & others & $11^{2}, 7^{4}, 3^{2}$ & $\left(A_{3}+A_{2}\right)^{(2)}$ & 2 & $7^{2}, 5^{6}, 3^{2}, 1^{6}$ \\
\hline & & & $A_{3}+A_{2}$ & 2 & $4^{8}, 3^{8}$ \\
\hline & & & & & $6^{2}, 5^{2}, 4^{2}, 3^{8}, 1^{2}$ \\
\hline & & & & & $5^{6}, 4^{2}, 3^{4}, 2^{2}, 1^{2}$ \\
\hline & & & & others & $6^{2}, 5^{2}, 4^{4}, 3^{4}, 2^{2}, 1^{2}$ \\
\hline
\end{tabular}


TABLE 3. Jordan blocks of nilpotent elements on the module $V_{56}$ for $E_{7}, I I$.

\begin{tabular}{|c|c|c|c|c|c|}
\hline$e$ & $p$ & Jordan blocks & $e$ & $p$ & Jordan blocks \\
\hline \multirow[t]{4}{*}{$D_{4}\left(a_{1}\right)+A_{1}$} & \multirow{4}{*}{$\begin{array}{l}2 \\
3 \\
5 \\
\text { others }\end{array}$} & \multirow{4}{*}{$\begin{array}{l}4^{12}, 2^{4} \\
6,5^{4}, 4,3^{6}, 2^{4} \\
5^{6}, 4,3^{4}, 2^{5} \\
6,5^{4}, 4^{2}, 3^{4}, 2^{5}\end{array}$} & \multirow[t]{3}{*}{$2 A_{2}$} & 2 & $4^{4}, 3^{12}, 1^{4}$ \\
\hline & & & & & $3^{18}, 1^{2}$ \\
\hline & & & & others & $5^{2}, 3^{14}, 1^{4}$ \\
\hline & & & \multirow[t]{2}{*}{$A_{3}$} & \multirow{2}{*}{$\begin{array}{l}2 \\
\text { others }\end{array}$} & \multirow{2}{*}{$\begin{array}{l}4^{8}, 3^{4}, 1^{12} \\
5^{2}, 4^{8}, 1^{14}\end{array}$} \\
\hline \multirow[t]{2}{*}{$D_{4}$} & \multirow{2}{*}{$\begin{array}{l}2 \\
\text { others }\end{array}$} & \multirow{2}{*}{$\begin{array}{l}4^{12}, 1^{8} \\
7^{6}, 1^{14}\end{array}$} & & & \\
\hline & & & \multirow{3}{*}{$A_{2}+2 A_{1}$} & 2 & $4^{4}, 3^{4}, 2^{12}, 1^{4}$ \\
\hline \multirow{3}{*}{$A_{3}+2 A_{1}$} & \multirow{3}{*}{$\begin{array}{l}2 \\
5 \\
\text { others }\end{array}$} & \multirow{3}{*}{$\begin{array}{l}4^{10}, 2^{8} \\
5^{4}, 4^{4}, 3^{4}, 2^{3}, 1^{2} \\
6,5^{2}, 4^{5}, 3^{4}, 2^{3}, 1^{2}\end{array}$} & & & $3^{14}, 2^{4}, 1^{6}$ \\
\hline & & & & others & $4^{4}, 3^{6}, 2^{8}, 1^{6}$ \\
\hline & & & \multirow{2}{*}{$A_{2}+A_{1}$} & \multirow{2}{*}{$\begin{array}{l}3 \\
\text { others }\end{array}$} & \multirow{2}{*}{$\begin{array}{l}3^{12}, 2^{6}, 1^{8} \\
4^{2}, 3^{8}, 2^{8}, 1^{8}\end{array}$} \\
\hline \multirow{2}{*}{$D_{4}\left(a_{1}\right)$} & \multirow{2}{*}{$\begin{array}{l}2 \\
\text { others }\end{array}$} & \multirow{2}{*}{$\begin{array}{l}4^{12}, 1^{8} \\
5^{6}, 3^{6}, 1^{8}\end{array}$} & & & \\
\hline & & & \multirow[t]{2}{*}{$4 A_{1}$} & 2 & $2^{28}$ \\
\hline$\left(A_{3}+A_{1}\right)^{\prime}$ & $\begin{array}{l}2 \\
\text { others }\end{array}$ & $\begin{array}{l}4^{8}, 3^{4}, 2^{4}, 1^{4} \\
5^{4}, 4^{4}, 3^{2}, 2^{4}, 1^{6}\end{array}$ & & $\begin{array}{l}3 \\
\text { others }\end{array}$ & $\begin{array}{l}3^{8}, 2^{13}, 1^{6} \\
4,3^{6}, 2^{14}, 1^{6}\end{array}$ \\
\hline \multirow[t]{2}{*}{$2 A_{2}+A_{1}$} & \multirow{2}{*}{$\begin{array}{l}2 \\
3 \\
\text { others } \\
\end{array}$} & \multirow{2}{*}{$\begin{array}{l}4^{8}, 3^{4}, 2^{4}, 1^{4} \\
3^{18}, 1^{2} \\
5^{2}, 4^{4}, 3^{6}, 2^{4}, 1^{4}\end{array}$} & $A_{2}$ & all & $3^{12}, 1^{20}$ \\
\hline & & & $\left(3 A_{1}\right)^{\prime}$ & $\begin{array}{l}2 \\
\text { others }\end{array}$ & $\begin{array}{l}2^{24}, 1^{8} \\
3^{6}, 2^{12}, 1^{14}\end{array}$ \\
\hline \multirow[t]{3}{*}{$\left(A_{3}+A_{1}\right)^{\prime \prime}$} & 2 & $4^{10}, 2^{8}$ & \multirow{3}{*}{$\left(3 A_{1}\right)^{\prime \prime}$} & & $2^{28}$ \\
\hline & & $5^{2}, 4^{8}, 2^{7}$ & & & $3^{2}, 2^{25}$ \\
\hline & others & $6,4^{9}, 2^{7}$ & & others & $4,2^{26}$ \\
\hline \multirow[t]{3}{*}{$A_{2}+3 A_{1}$} & \multirow{3}{*}{$\begin{array}{l}3 \\
\text { others } \\
\end{array}$} & \multirow{3}{*}{$\begin{array}{l}4^{6}, 2^{16} \\
3^{14}, 2^{7} \\
4^{7}, 2^{14} \\
\end{array}$} & \multirow[t]{2}{*}{$2 A_{1}$} & \multirow{2}{*}{$\begin{array}{l}2 \\
\text { others }\end{array}$} & \multirow{2}{*}{$\begin{array}{l}2^{20}, 1^{16} \\
3^{2}, 2^{16}, 1^{18}\end{array}$} \\
\hline & & & & & \\
\hline & & & $A_{1}$ & all & $2^{12}, 1^{32}$ \\
\hline
\end{tabular}


TABLE 4. Jordan blocks of nilpotent elements on the module $V_{27}$ for $E_{6}, V_{26}$ for $F_{4}$ and $V_{7}$ for $G_{2}$.

\begin{tabular}{|c|c|c|c|c|c|c|c|c|}
\hline$e$ & $p$ & Jordan blocks & $e$ & $p$ & Jordan blocks & $e$ & $p$ & Jordan blocks \\
\hline \multirow[t]{4}{*}{$E_{6}$} & 2 & \multirow{4}{*}{$\begin{array}{l}13^{2}, 1 \\
9^{3} \\
13^{2}, 1 \\
17,9,1\end{array}$} & \multirow[t]{4}{*}{$F_{4}$} & \multirow{4}{*}{$\begin{array}{l}2 \\
3 \\
13 \\
\text { others }\end{array}$} & \multirow{4}{*}{$\begin{array}{l}13^{2} \\
9^{2}, 8 \\
13^{2} \\
17,9 \\
\end{array}$} & \multirow[t]{2}{*}{$G_{2}$} & & \multirow{2}{*}{$\begin{array}{l}4,3 \\
7\end{array}$} \\
\hline & \multirow{3}{*}{$\begin{array}{l}3 \\
13 \\
\text { others }\end{array}$} & & & & & & others & \\
\hline & & & & & & $G_{2}\left(a_{1}\right)$ & all & $3^{2}, 1$ \\
\hline & & & & & & $\left(\tilde{A}_{1}\right)^{(3)}$ & 3 & $3,2^{2}$ \\
\hline \multirow{3}{*}{$E_{6}\left(a_{1}\right)$} & \multirow{3}{*}{$\begin{array}{l}3 \\
7 \\
11 \\
\text { others }\end{array}$} & $9^{3}$ & \multirow{2}{*}{$\overline{F_{4}\left(a_{1}\right)}$} & \multirow{2}{*}{$\begin{array}{l}2 \\
3 \\
\text { others }\end{array}$} & \multirow{2}{*}{$\begin{array}{l}8^{2}, 5^{2} \\
9,8^{2}, 1 \\
11,9,5,1\end{array}$} & & & \\
\hline & & $13,7^{2}$ & & & & $A_{1}$ & $\begin{array}{l}2 \\
\text { others }\end{array}$ & $\begin{array}{l}2^{3}, 1 \\
3,2^{2}\end{array}$ \\
\hline & & $13,9,5$ & \multirow{4}{*}{$\overline{F_{4}\left(a_{2}\right)}$} & 2 & $\frac{11,5,0,1}{8^{2}, 5^{2}}$ & $A_{1}$ & all & $2^{2}, 1^{3}$ \\
\hline \multirow[t]{3}{*}{$D_{5}$} & 2 & $8^{2}, 5^{2}, 1$ & & 3 & $9,6^{2}, 3,2$ & & & \\
\hline & 3 & $9,8^{2}, 1^{2}$ & & 7 & $7^{3}, 5$ & & & \\
\hline & others & $11,9,5,1^{2}$ & & others & $9,7,5^{2}$ & & & \\
\hline \multirow[t]{4}{*}{$E_{6}\left(a_{3}\right)$} & \multirow{4}{*}{$\begin{array}{l}2 \\
3 \\
7 \\
\text { others }\end{array}$} & \multirow{4}{*}{$\begin{array}{l}8^{2}, 5^{2}, 1 \\
9,6^{2}, 3^{2} \\
7^{3}, 5,1 \\
9,7,5^{2}, 1\end{array}$} & $\left(C_{3}\right)^{(2)}$ & 2 & $7^{2}, 6^{2}$ & & & \\
\hline & & & \multirow[t]{4}{*}{$C_{3}$} & 2 & $7^{2}, 6^{2}$ & & & \\
\hline & & & & 3 & $9,6^{2}, 3,2$ & & & \\
\hline & & & & & $7^{2}, 6^{2}$ & & & \\
\hline \multirow[t]{3}{*}{$D_{5}\left(a_{1}\right)$} & \multirow{3}{*}{$\begin{array}{l}2 \\
7 \\
\text { others }\end{array}$} & \multirow{3}{*}{$\begin{array}{l}8,5^{2}, 4^{2}, 1 \\
7^{3}, 3,2,1 \\
8,7,6,3,2,1\end{array}$} & & others & $9,6^{2}, 5$ & & & \\
\hline & & & $B_{3}$ & 2 & $4^{6}, 1^{2}$ & & & \\
\hline & & & & others & $7^{3}, 1^{5}$ & & & \\
\hline$A_{5}$ & 2 & $7^{2}, 6^{2}, 1$ & $F_{4}\left(a_{3}\right)$ & & $4^{6}, 1^{2}$ & & & \\
\hline & & $9,6^{2}, 3^{2}$ & & others & $5^{3}, 3^{3}, 1^{2}$ & & & \\
\hline & others & $\begin{array}{l}7^{2}, 6^{2}, 1 \\
9,6^{2}, 5,1\end{array}$ & $C_{3}\left(a_{1}\right)^{(2)}$ & 2 & $4^{4}, 3^{2}, 2^{2}$ & & & \\
\hline$A_{4}+A_{1}$ & $\begin{array}{l}5 \\
\text { others }\end{array}$ & $\begin{array}{l}5^{5}, 2 \\
76,5,32\end{array}$ & $C_{3}\left(a_{1}\right)$ & $\begin{array}{l}2 \\
\text { others }\end{array}$ & $\begin{array}{l}4^{4}, 3^{2}, 2^{2} \\
5^{2}, 4^{2}, 3,2^{2}, 1\end{array}$ & & & \\
\hline$D_{4}$ & ouners & $\frac{1,0,5,4,3,2}{4^{6}, 1^{3}}$ & $\left(\tilde{A}_{2}+A_{1}\right)^{(2)}$ & 2 & $4^{4}, 3^{2}, 2^{2}$ & & & \\
\hline & others & $\begin{array}{l}7^{3}, 1^{6} \\
\end{array}$ & $\tilde{A}_{2}+A_{1}$ & 2 & $4^{4}, 3^{2}, 2^{2}$ & & & \\
\hline$A_{4}$ & $\begin{array}{l}5 \\
\text { others }\end{array}$ & $\begin{array}{l}5^{5}, 1^{2} \\
7,5^{3} 31^{2}\end{array}$ & & $\begin{array}{l}3 \\
\text { others }\end{array}$ & $\begin{array}{l}3^{8}, 2 \\
5,4^{2}, 3^{3}, 2^{2}\end{array}$ & & & \\
\hline$D_{4}\left(a_{1}\right)$ & $\begin{array}{l}\text { otners } \\
2\end{array}$ & $\frac{7,5^{5}, 3,1^{2}}{4^{6}, 1^{3}}$ & $\left(B_{2}\right)^{(2)}$ & 2 & $4^{4}, 3^{2}, 1^{4}$ & & & \\
\hline$D_{4}\left(a_{1}\right)$ & $\begin{array}{l}2 \\
\text { others }\end{array}$ & $5^{3}, 3^{3}, 1^{3}$ & $B_{2}$ & & $4^{4}, 3^{2}, 1^{4}$ & & & \\
\hline$A_{3}+A_{1}$ & 2 & $4^{4}, 3^{2}, 2^{2}, 1$ & & others & $5,4^{4}, 1^{5}$ & & & \\
\hline & others & $5^{2}, 4^{2}, 3,2^{2}, 1^{2}$ & $\overline{A_{2}+\tilde{A}_{1}}$ & 2 & $4^{2}, 3^{2}, 2^{6}$ & & & \\
\hline $2 A_{2}+A_{1}$ & 2 & $4^{4}, 3^{2}, 2^{2}, 1$ & & $\begin{array}{l}3 \\
\text { others }\end{array}$ & $\begin{array}{l}3^{7}, 2^{2}, 1 \\
4^{2}, 3^{3}, 2^{4}, 1\end{array}$ & & & \\
\hline & $\begin{array}{l}3 \\
\text { others }\end{array}$ & $5,4^{2}, 3^{3}, 2^{2}, 1$ & $\tilde{A}_{2}$ & 2 & $4^{2}, 3^{6}$ & & & \\
\hline$A_{3}$ & 2 & $4^{4}, 3^{2}, 1^{5}$ & & $\begin{array}{l}3 \\
\text { others }\end{array}$ & $\begin{array}{l}3^{8}, 2 \\
5,3^{7}\end{array}$ & & & \\
\hline & others & $\frac{5,4^{4}, 1^{6}}{4^{2} 2^{2} 2^{6}}$ & $\overline{\left(A_{2}\right)^{(2)}}$ & 2 & $3^{6}, 1^{8}$ & & & \\
\hline$A_{2}+2 A_{1}$ & $\begin{array}{l}2 \\
3\end{array}$ & $\begin{array}{l}4^{2}, 3^{2}, 2^{6}, 1 \\
3^{7}, 2^{2}, 1^{2}\end{array}$ & $A_{2}$ & all & $3^{6}, 1^{8}$ & & & \\
\hline & others & $4^{2}, 3^{3}, 2^{4}, 1^{2}$ & $\overline{A_{1}+\tilde{A}_{1}}$ & & $2^{12}, 1^{2}$ & & & \\
\hline $2 A_{2}$ & 2 & $4^{2}, 3^{6}, 1$ & & others & $3^{3}, 2^{6}, 1^{5}$ & & & \\
\hline & & $3^{9}$ & $\overline{\left(\tilde{A}_{1}\right)^{(2)}}$ & 2 & $2^{10}, 1^{6}$ & & & \\
\hline & others & $5,3^{7}, 1$ & $\tilde{A}_{1}$ & & $2^{10}, 1^{6}$ & & & \\
\hline$A_{2}+A_{1}$ & 3 & $3^{6}, 2^{3}, 1^{3}$ & & others & $3,2^{8}, 1^{7}$ & & & \\
\hline & others & $4,3^{4}, 2^{4}, 1^{3}$ & $A_{1}$ & all & $2^{6}, 1^{14}$ & & & \\
\hline$A_{2}$ & all & $3^{6}, 1^{9}$ & & & & & & \\
\hline $3 A_{1}$ & $\begin{array}{l}2 \\
\text { others }\end{array}$ & $\begin{array}{l}2^{12}, 1^{3} \\
3^{3}, 2^{6}, 1^{6}\end{array}$ & & & & & & \\
\hline $2 A_{1}$ & $\begin{array}{l}2 \\
\text { others }\end{array}$ & $\begin{array}{l}2^{10}, 1^{7} \\
3,2^{8}, 1^{8}\end{array}$ & & & & & & \\
\hline$A_{1}$ & all & $2^{6}, 1^{15}$ & & & & & & \\
\hline
\end{tabular}




\section{Appendix}

\section{A.1. Representatives of nilpotent orbits}

TABle A.1. Representatives of nilpotent elements for $G_{2}$ and $F_{4}$.

\begin{tabular}{|c|c|c|c|}
\hline Orbit & Representative & Orbit & Representative \\
\hline$G_{2}$ & $e_{10}+e_{01}$ & $F_{4}$ & $e_{0001}+e_{1000}+e_{0010}+e_{0100}$ \\
\hline$G_{2}\left(a_{1}\right)$ & $e_{01}+e_{31}$ & $F_{4}\left(a_{1}\right)$ & $e_{1000}+e_{0100}+e_{0011}+e_{0110}$ \\
\hline$\left(\tilde{A}_{1}\right)^{(3)}$ & $e_{21}+e_{32}$ & $F_{4}\left(a_{2}\right)$ & $e_{0001}+e_{0011}+e_{1100}+e_{0120}$ \\
\hline$\tilde{A}_{1}$ & $e_{10}$ & $\left(C_{3}\right)^{(2)}$ & $e_{0001}+e_{1110}+e_{0120}+e_{1222}$ \\
\hline \multirow[t]{17}{*}{$A_{1}$} & $e_{01}$ & $C_{3}$ & $e_{0001}+e_{0010}+e_{0100}$ \\
\hline & & $B_{3}$ & $e_{1000}+e_{0010}+e_{0100}$ \\
\hline & & $F_{4}\left(a_{3}\right)$ & $e_{0100}+e_{1100}+e_{0120}+e_{1122}$ \\
\hline & & $C_{3}\left(a_{1}\right)^{(2)}$ & $e_{1110}+e_{0120}+e_{0121}+e_{1222}$ \\
\hline & & $C_{3}\left(a_{1}\right)$ & $e_{0100}+e_{0011}+e_{0120}$ \\
\hline & & $\left(\tilde{A}_{2}+A_{1}\right)^{(2)}$ & $e_{0111}+e_{1121}+e_{0122}+e_{1220}$ \\
\hline & & $\tilde{A}_{2}+A_{1}$ & $e_{0001}+e_{1000}+e_{0010}$ \\
\hline & & $\left(B_{2}\right)^{(2)}$ & $e_{1100}+e_{1120}+e_{0122}$ \\
\hline & & $B_{2}$ & $e_{0010}+e_{0100}$ \\
\hline & & $A_{2}+\tilde{A}_{1}$ & $e_{0001}+e_{1000}+e_{0100}$ \\
\hline & & $\tilde{A}_{2}$ & $e_{0001}+e_{0010}$ \\
\hline & & $\left(A_{2}\right)^{(2)}$ & $e_{1220}+e_{1122}$ \\
\hline & & $A_{2}$ & $e_{1000}+e_{0100}$ \\
\hline & & $A_{1}+\tilde{A}_{1}$ & $e_{1000}+e_{0010}$ \\
\hline & & $\left(\tilde{A}_{1}\right)^{(2)}$ & $e_{1232}+e_{2342}$ \\
\hline & & $\tilde{A}_{1}$ & $e_{0010}$ \\
\hline & & $A_{1}$ & $e_{1000}$ \\
\hline
\end{tabular}

TABle A.2. Representatives of nilpotent elements for $E_{6}$.

\begin{tabular}{|c|c|}
\hline Orbit & Representative \\
\hline$E_{6}$ & $e_{0} 0000+e_{00000}+e_{01000}+e_{00100}+e_{00010}+e_{00001}$ \\
\hline$E_{6}\left(a_{1}\right)$ & $e_{0}^{10000}+\underset{0}{e_{01000}}+e_{0}^{00010}+e_{00001}+e_{00100}+e_{01100}$ \\
\hline$D_{5}$ & $e_{0}^{10000}+e_{1}^{00000}+\underset{0}{e 01000}+e_{0}^{000}+e_{0}^{00010}$ \\
\hline$E_{6}\left(a_{3}\right)$ & $e_{10000}+e_{00100}+e_{01100}+e_{00011}+e_{00110}+e_{01110}$ \\
\hline$D_{5}\left(a_{1}\right)$ & $e_{00000}+e_{00010}^{0}+e_{00001}^{0}+e_{00100}+e_{01100}$ \\
\hline$A_{5}$ & $e_{0}^{10000}+\underset{0}{e_{01000}}+e_{0}^{00100}+e_{0}^{00010}+e_{0001}^{0000}$ \\
\hline$A_{4}+A_{1}$ & $e_{0} \underset{000}{ }+e_{1}^{00000}+\underset{0}{e_{01000}}+e_{00100}^{0}+e_{00001}$ \\
\hline$D_{4}$ & $e_{00000}+\underset{0}{e_{01000}}+e_{0}^{00100}+\underset{0}{e_{00010}}$ \\
\hline$A_{4}$ & $e_{0} \underset{0000}{e}+\underset{0}{e} 000+e_{0}^{00100}+e_{00010}^{000}$ \\
\hline$D_{4}\left(a_{1}\right)$ & $e_{1}^{00000}+\underset{0}{e 01000}+\underset{0}{e 01100}+e_{00110}$ \\
\hline$A_{3}+A_{1}$ & $e_{0}^{10000}+\underset{0}{e_{01000}}+e_{00100}+e_{00001}$ \\
\hline $2 A_{2}+A_{1}$ & $e_{0} \underset{000}{0}+\underset{1}{e 0000}+\underset{0}{e 01000}+\underset{0}{e 00010}+e_{0001}^{00001}$ \\
\hline$A_{3}$ & $e_{10000}+e_{01000}+e_{00100}$ \\
\hline$A_{2}+2 A_{1}$ & $e_{0} \underset{0000}{0}+\underset{1}{e_{00000}}+e_{01000}+e_{00001}$ \\
\hline $2 A_{2}$ & $e_{0}^{10000}+\underset{0}{e_{01000}}+e_{00010}+e_{00001}$ \\
\hline$A_{2}+A_{1}$ & $e_{10000}+e_{01000}+e_{00010}$ \\
\hline$A_{2}$ & $e_{10000}+\underset{0}{e_{01000}}$ \\
\hline $3 A_{1}$ & $e_{0}^{10000}+\underset{0}{e_{00100}}+e_{00001}$ \\
\hline $2 A_{1}$ & $e_{10000}+e_{00100}$ \\
\hline$A_{1}$ & $e_{10000}$ \\
\hline
\end{tabular}


TABle A.3. Representatives of nilpotent elements for $E_{7}$.

\begin{tabular}{|c|c|}
\hline Orbit & Representative \\
\hline$E_{7}$ & $e_{100000}+e_{000000}+e_{010000}+e_{001000}+e_{000100}+e_{000010}+e_{000001}$ \\
\hline$E_{7}\left(a_{1}\right)$ & $e_{0} \underset{0}{00000}+e_{0}^{010000}+e_{0}^{0000100}+e_{0}^{0000010}+e_{0}^{0000001}+e_{0}^{001000}+e_{0}^{011000}$ \\
\hline$E_{7}\left(a_{2}\right)$ & $e_{0} \underset{0}{10000}+e_{1}^{000000}+e_{0}^{010000}+e_{1}^{001000}+e_{0}^{0001100}+e_{0}^{000110}+e_{0}^{000011}$ \\
\hline$E_{7}\left(a_{3}\right)$ & $e_{0} \underset{0}{00000}+e_{0}^{000001}+e_{001000}+e_{011000}+e_{0}^{000110}+e_{001100}+e_{011100}$ \\
\hline$E_{6}$ & $e_{0} e_{0} 000+e_{00000}+e_{010000}+e_{001000}+e_{0}^{000100}+e_{0}^{000010}$ \\
\hline$E_{6}\left(a_{1}\right)$ & $e_{0}^{100000}+e_{0}^{010000}+\underset{0}{e 000100}+e_{0}^{0000010}+e_{1}^{001000}+e_{0}^{011000}$ \\
\hline$D_{6}$ & $e_{1}^{0000000}+\underset{0}{e 010000}+e_{0}^{0001000}+e_{0}^{0000100}+e_{0}^{000010}+e_{0}^{000001}$ \\
\hline$E_{7}\left(a_{4}\right)$ & $e_{100000}+e_{000011}+e_{011000}+e_{001100}+e_{011100}+e_{001110}+e_{0}^{011110}$ \\
\hline$D_{6}\left(a_{1}\right)$ & $e_{000000}+e_{000100}+e_{0}^{000010}+e_{0}^{000001}+e_{001000}+e_{011000}$ \\
\hline$D_{5}+A_{1}$ & $e_{0}^{100000}+e_{1}^{000000}+\underset{0}{e 010000}+e_{0}^{0001000}+e_{0}^{000100}+e_{0}^{000001}$ \\
\hline$\left(A_{6}\right)^{(2)}$ & $e_{000110}+e_{000011}+e_{111000}+e_{011000}+e_{001100}+e_{011100}+e_{122100}$ \\
\hline$A_{6}$ & $e_{0} \underset{0}{10000}+\underset{0}{e 010000}+e_{0}^{001000}+e_{0}^{0000100}+e_{0}^{0000010}+e_{0}^{000001}$ \\
\hline$E_{7}\left(a_{5}\right)$ & $e_{0}^{111000}+e_{011000}^{0}+e_{1}^{001100}+e_{0}^{001110}+e_{0}^{000111}+e_{0}^{011110}+e_{111110}$ \\
\hline$D_{5}$ & $e_{0} \underset{0}{10000}+e_{1}^{000000}+\underset{0}{e 010000}+e_{0}^{0001000}+e_{0}^{0000100}$ \\
\hline$E_{6}\left(a_{3}\right)$ & $e_{0} \underset{0}{100000}+e_{0}^{000010}+e_{0} \underset{0}{110000}+e_{1}^{0001000}+e_{0}^{000110}+e_{0}^{011100}$ \\
\hline$D_{6}\left(a_{2}\right)$ & $e_{1}^{000000}+e_{0}^{010000}+e_{0}^{011000}+e_{0}^{001100}+e_{0}^{0000110}+e_{0}^{000011}$ \\
\hline$D_{5}\left(a_{1}\right)+A_{1}$ & $e_{0}^{100000}+e_{0}^{010000}+e_{0}^{0000100}+e_{0}^{000001}+e_{1}^{0001000}+e_{0}^{001100}$ \\
\hline$A_{5}+A_{1}$ & $e_{0}^{100000}+\underset{1}{e 000000}+\underset{0}{e 001000}+e_{0}^{0000100}+e_{0}^{0000010}+e_{0}^{000001}$ \\
\hline$\left(A_{5}\right)^{\prime}$ & $e_{0}^{100000}+\underset{0}{e 010000}+e_{0}^{0001000}+e_{0}^{0000100}+e_{0}^{0000010}$ \\
\hline$A_{4}+A_{2}$ & $e_{0} \underset{0}{0}+\underset{1}{e 000000}+\underset{0}{e 010000}+\underset{0}{e 001000}+e_{0}^{0000010}+\underset{0}{e 00001}$ \\
\hline$D_{5}\left(a_{1}\right)$ & $e_{0} \underset{0}{0000}+e_{010000}^{0}+e_{0}^{0000100}+e_{001000}^{00}+e_{0}^{001100}$ \\
\hline$A_{4}+A_{1}$ & $e_{0} \underset{0}{0} 0000+e_{0}^{010000}+\underset{0}{e 001000}+e_{0}^{000100}+e_{0}^{0000001}$ \\
\hline$D_{4}+A_{1}$ & $e_{000000}+e_{0}^{010000}+e_{0}^{0001000}+e_{0}^{000100}+e_{0}^{000001}$ \\
\hline$\left(A_{5}\right)^{\prime \prime}$ & $e_{1}^{000000}+e_{0}^{0001000}+e_{0}^{0000100}+e_{0}^{0000010}+e_{0}^{000001}$ \\
\hline$A_{3}+A_{2}+A_{1}$ & $e_{0}^{100000}+e_{1}^{000000}+\underset{0}{e 010000}+\underset{0}{e 000100}+e_{0}^{0000010}+e_{0}^{000001}$ \\
\hline$A_{4}$ & $e_{0}^{100000}+\underset{0}{e 010000}+\underset{0}{e 001000}+e_{0}^{0000100}$ \\
\hline$\left(A_{3}+A_{2}\right)^{(2)}$ & $e_{0}^{000001}+e_{1}^{001000}+e_{0}^{000011}+e_{0}^{011100}+e_{0}^{001110}+e_{011110}$ \\
\hline$A_{3}+A_{2}$ & $e_{100000}+e_{010000}^{0}+e_{0}^{001000}+e_{000010}+e_{00001}^{0000}$ \\
\hline$D_{4}\left(a_{1}\right)+A_{1}$ & $e_{1}^{000000}+\underset{0}{0010000}+e_{0}^{0000001}+e_{1}^{0001000}+e_{0}^{001100}$ \\
\hline$D_{4}$ & $e_{1}^{e 000000}+\underset{0}{e 010000}+\underset{0}{e 001000}+\underset{0}{\operatorname{expop} 100}$ \\
\hline$A_{3}+2 A_{1}$ & $e_{0} \underset{0}{10000}+e_{1}^{000000}+\underset{0}{e 001000}+\underset{0}{0000100}+e_{0}^{000001}$ \\
\hline$D_{4}\left(a_{1}\right)$ & $\underset{1}{e 000000}+\underset{0}{e 010000}+\underset{1}{e 001000}+\underset{0}{e 001100}$ \\
\hline$\left(A_{3}+A_{1}\right)^{\prime}$ & $e_{0}^{100000}+\underset{0}{e 010000}+e_{0}^{0001000}+e_{0}^{000010}$ \\
\hline $2 A_{2}+A_{1}$ & $e_{0} \underset{0}{10000}+e_{000000}^{0}+e_{0}^{010000}+e_{0}^{000100}+e_{0}^{000010}$ \\
\hline$\left(A_{3}+A_{1}\right)^{\prime \prime}$ & $e_{1}^{000000}+\underset{0}{e 000100}+\underset{0}{e 000010}+e_{0}^{000001}$ \\
\hline$A_{2}+3 A_{1}$ & $e_{0}^{100000}+e_{1}^{000000}+\underset{0}{e 010000}+e_{0}^{0000100}+e_{0}^{000001}$ \\
\hline $2 A_{2}$ & $e_{0} \underset{0}{10000}+\underset{0}{e 010000}+\underset{0}{e 000010}+e_{0}^{000001}$ \\
\hline$A_{3}$ & $e_{0}^{100000}+\underset{0}{e 010000}+\underset{0}{e 001000}$ \\
\hline$A_{2}+2 A_{1}$ & $e_{0} \underset{0}{10000}+\underset{0}{e 010000}+\underset{0}{e 000100}+e_{00001}^{0000}$ \\
\hline$A_{2}+A_{1}$ & $e_{0} \underset{0}{10000}+\underset{0}{0010000}+e_{0}^{0000100}$ \\
\hline
\end{tabular}




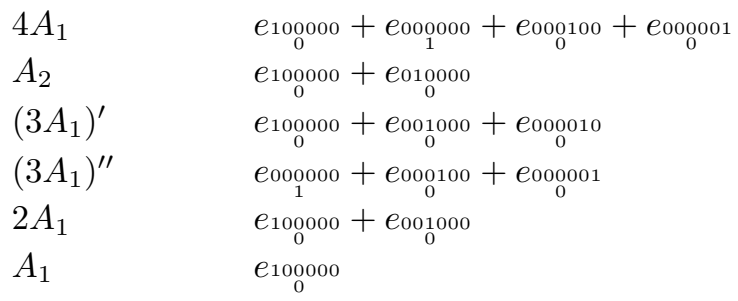

TABlE A.4. Representatives of nilpotent elements for $E_{8}$.

\begin{tabular}{|c|c|}
\hline Orbit & Representative \\
\hline$E_{8}$ & $e_{100000}+e_{0000000}+e_{0100000}+e_{0}^{0010000}+e_{0}^{0001000}+e_{0000100}+e_{0}^{0000010}+e_{0000001}$ \\
\hline$E_{8}\left(a_{1}\right)$ & $e_{0}^{1000000}+\underset{1}{e 0000000}+\underset{0}{e 0001000}+\underset{0}{e 0000100}+\underset{0}{e 0000010}+e_{0}^{00000001}+e_{1}^{00010000}+e_{0}^{00110000}$ \\
\hline$E_{8}\left(a_{2}\right)$ & $e_{0}^{1000000}+e_{1}^{00000000}+\underset{0}{e 0100000}+\underset{0}{e 0000001}+e_{1}^{00010000}+e_{0}^{00011000}+e_{0}^{00001100}+e_{0}^{00000110}$ \\
\hline$E_{8}\left(a_{3}\right)$ & $e_{0}^{00000010}+\underset{0}{00000001}+e_{0} \underset{0}{00000}+e_{1}^{00010000}+e_{0}^{0110000}+e_{0}^{00011000}+e_{0}^{00001100}+e_{0}^{00111000}$ \\
\hline$E_{8}\left(a_{4}\right)$ & $e_{0}^{1100000}+e \underset{1}{00010000}+e_{0}^{0110000}+e_{0}^{00011000}+e_{0}^{00001100}+e_{0}^{0000110}+e_{0}^{00000011}+e_{0}^{00111000}$ \\
\hline$E_{7}$ & $e_{0}^{100000}+\underset{1}{e 0000000}+\underset{0}{e 0100000}+\underset{0}{e 0010000}+\underset{0}{e 0001000}+e_{0}^{00000100}+e_{0}^{00000010}$ \\
\hline$E_{8}\left(b_{4}\right)$ & $e_{0}^{00000010}+e_{0}^{0000001}+e_{0}^{e 1100000}+e_{1}^{00110000}+e_{1}^{00011000}+e_{0}^{0111000}+e_{0}^{00011100}+e_{0}^{0001110}$ \\
\hline$E_{8}\left(a_{5}\right)$ & $e_{0}^{1100000}+e \underset{0}{0000110}+e_{0}^{0000011}+e_{1}^{0110000}+e_{1}^{0011000}+e_{0}^{0111000}+e_{0}^{0011100}+e_{1}^{00011100}$ \\
\hline$E_{7}\left(a_{1}\right)$ & $e_{0}^{1000000}+\underset{0}{e 0100000}+\underset{0}{e 0001000}+\underset{0}{e 0000100}+\underset{0}{e 0000010}+e_{1}^{0010000}+e_{0}^{0110000}$ \\
\hline$E_{8}\left(b_{5}\right)$ & $e_{0}^{0000001}+e_{1110000}+e_{0110000}+e_{1}^{0011000}+e_{0}^{0011100}+e_{0001110}+e_{1111000}+e_{0}^{0111100}$ \\
\hline$\left(D_{7}\right)^{(2)}$ & $e_{0}^{100000}+e_{1}^{0110000}+e_{1}^{0011000}+e_{0}^{0111000}+e_{0}^{00011100}+e_{0}^{0001110}+e_{0}^{0000111}+e_{1111111}$ \\
\hline$D_{7}$ & $e_{1}^{00000000}+\underset{0}{00100000}+e_{0}^{00010000}+e_{0}^{00001000}+\underset{0}{e 0000100}+e_{0}^{00000010}+e_{0}^{00000001}$ \\
\hline$E_{8}\left(a_{6}\right)$ & $e_{0}^{0000110}+e_{0}^{0000011}+e_{1110000}+e_{0}^{0011100}+e_{1110000}+e_{1111000}+e_{0111000}+e_{1111100}$ \\
\hline$E_{7}\left(a_{2}\right)$ & $e_{0} \underset{0}{100000}+\underset{1}{e 0000000}+\underset{0}{e 0100000}+e_{1}^{00010000}+\underset{0}{e 0011000}+e_{0}^{0001100}+e_{0}^{00000110}$ \\
\hline$E_{6}+A_{1}$ & $e_{0} \underset{0}{100000}+e_{1}^{00000000}+e_{0}^{0100000}+\underset{0}{e 0010000}+\underset{0}{e 0001000}+e_{0}^{0000100}+e_{0}^{0000001}$ \\
\hline$\left(D_{7}\left(a_{1}\right)\right)^{(2)}$ & $e_{0}^{00001000}+\underset{0}{00000001}+e_{1100000}+e_{0}^{00011000}+\underset{0}{e 0000011}+e_{0}^{0011100}+e_{0}^{0111100}+e_{0121110}^{012}$ \\
\hline$D_{7}\left(a_{1}\right)$ & $e_{1}^{00000000}+\underset{0}{e 0001000}+\underset{0}{00000100}+\underset{0}{e 0000010}+\underset{0}{0000001}+e_{1}^{00010000}+e_{0}^{00110000}$ \\
\hline$E_{8}\left(b_{6}\right), p>2$ & $\underset{0}{e 0000011}+\underset{0}{e 0001111}+e_{1}^{e} \underset{1}{0} 0000+e_{0}^{1111000}+e_{1}^{00111000}+e_{1}^{00011100}+e_{0}^{0111100}+e_{0}^{00011110}$ \\
\hline$E_{8}\left(b_{6}\right), p=2$ & $e_{0}^{00000011}+e_{0}^{0000111}+e_{1110000}+e_{1}^{1111000}+e_{1}^{0111000}+e_{1}^{0011100}+e_{0}^{0111100}+e_{0}^{00011110}$ \\
\hline$E_{7}\left(a_{3}\right)$ & $e_{0} \underset{0}{00000}+e_{0}^{00000010}+e_{0010000}^{00}+e_{0}^{0110000}+e_{0}^{00001100}+e_{0}^{0011000}+e_{1}^{0111000}$ \\
\hline$E_{6}\left(a_{1}\right)+A_{1}$ & $e_{1000000}+e_{0}^{0100000}+e_{0}^{0001000}+e_{0}^{0000100}+e_{0}^{0000001}+e_{1}^{0010000}+e_{0}^{0110000}$ \\
\hline$A_{7}^{(3)}$ & $e_{0}^{00001110}+e_{0}^{0000111}+e_{1110000}+e_{1111000}+e_{0}^{0011100}+e_{0111100}+e_{0121000}+e_{0}^{0011111}$ \\
\hline$A_{7}$ & $e_{0}^{1000000}+\underset{0}{e 0100000}+\underset{0}{e 0010000}+e_{0}^{00001000}+e_{0}^{0000100}+e_{0}^{00000010}+e_{0}^{00000001}$ \\
\hline$D_{7}\left(a_{2}\right)$ & $e_{1}^{00000000}+\underset{0}{e 0100000}+\underset{0}{e 0000001}+\underset{0}{e 0110000}+\underset{0}{e 0011000}+\underset{0}{e 0001100}+\underset{0}{e 0000110}$ \\
\hline$E_{6}$ & $e_{0}^{1000000}+\underset{1}{e 0000000}+\underset{0}{e 0100000}+\underset{0}{e 0010000}+\underset{0}{e 0001000}+\underset{0}{00000100}$ \\
\hline$D_{6}$ & $e_{1}^{00000000}+\underset{0}{e 010000}+\underset{0}{e 0010000}+\underset{0}{e 0001000}+\underset{0}{e 0000100}+\underset{0}{e 0000010}$ \\
\hline$\left(D_{5}+A_{2}\right)^{(2)}$ & $e_{0}^{00000011}+e_{0}^{0000111}+e_{1111000}+e_{1111100}+e_{0121000}+e_{0111100}+e_{1}^{0011110}+e_{0111110}^{0}$ \\
\hline$D_{5}+A_{2}$ & $e_{0}^{1000000}+\underset{1}{e 0000000}+\underset{0}{e 0100000}+e_{0}^{00010000}+e_{0}^{00001000}+e_{0}^{00000010}+e_{0}^{00000001}$ \\
\hline$E_{6}\left(a_{1}\right)$ & $e_{0} \underset{0}{1000000}+e_{0}^{0010000}+e_{0}^{00001000}+e_{0}^{0000100}+e_{1}^{0010000}+e_{0}^{0110000}$ \\
\hline$E_{7}\left(a_{4}\right)$ & $e_{0} \underset{0}{00000}+e_{0000110}^{000}+e_{0110000}+e_{10011000}^{0}+e_{0111000}+e_{0011100}+e_{0111100}$ \\
\hline$A_{6}+$ & $e_{0} \underset{0}{0} 0000+\underset{1}{e 0000000}+\underset{0}{e 0010000}+\underset{0}{e 0001000}+\underset{0}{e 0000100}+\underset{0}{e 0000010}+\underset{0}{e 000001}$ \\
\hline
\end{tabular}




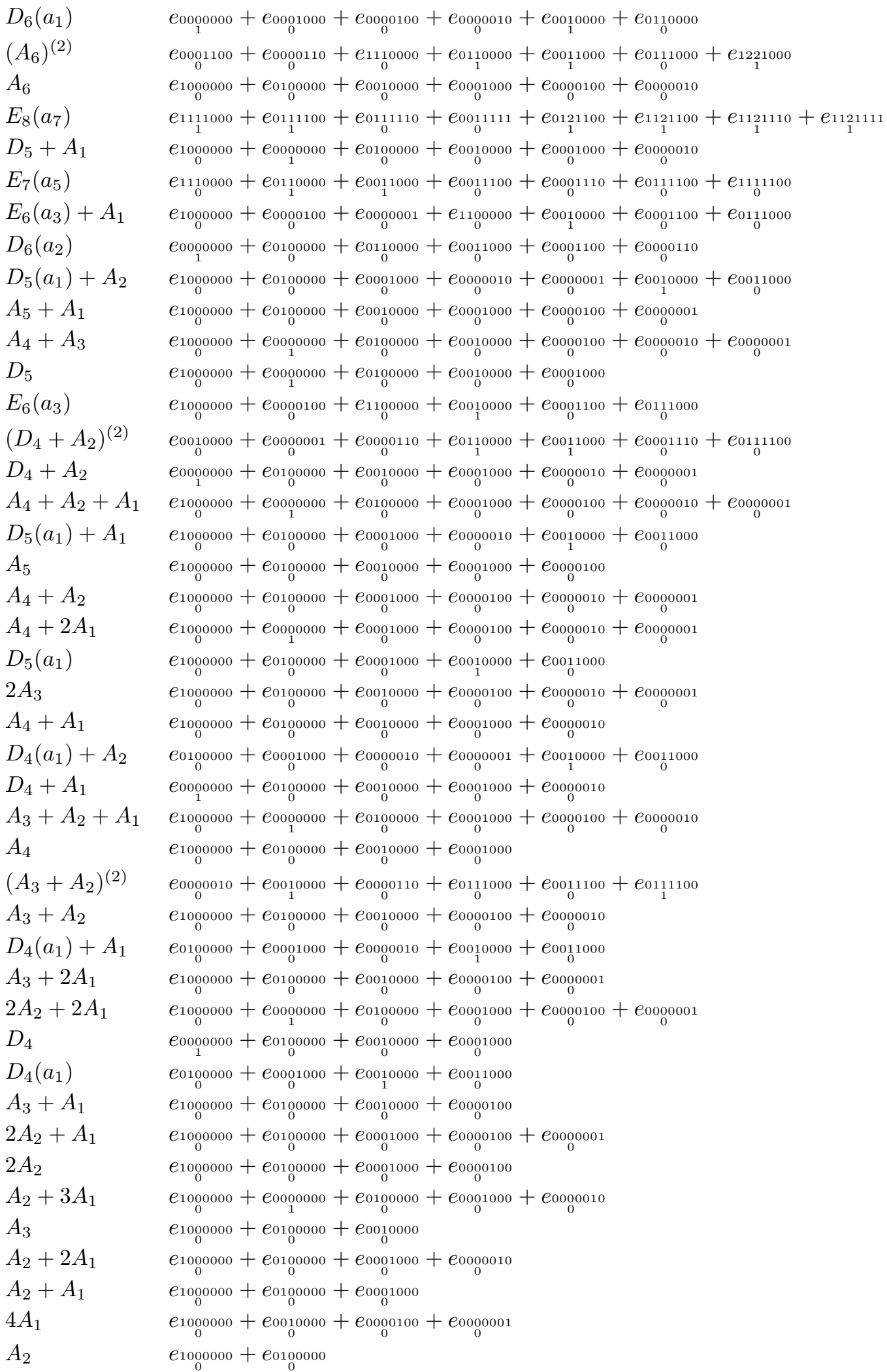




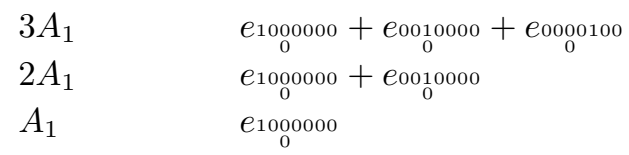

\section{A.2. Adjoint Jordan blocks}

TABLE A.5. Jordan blocks of nilpotent elements on the adjoint module for $E_{8}, I$.

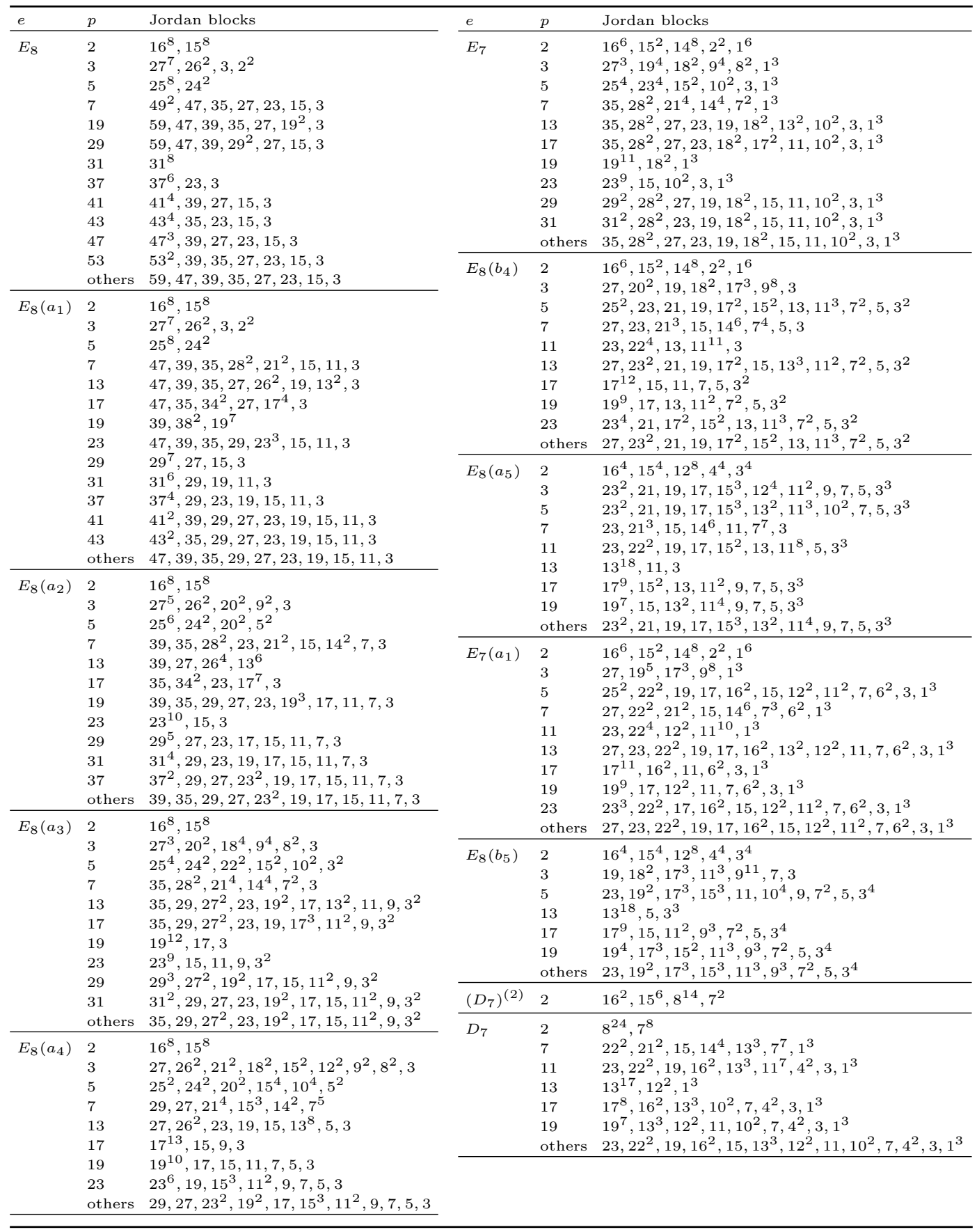


TABLE A.6. Jordan blocks of nilpotent elements on the adjoint module for $E_{8}$, II.

\begin{tabular}{|c|c|c|}
\hline$e$ & $p$ & Jordan blocks \\
\hline$E_{8}\left(a_{6}\right)$ & $\begin{array}{l}2 \\
3 \\
5 \\
7 \\
11 \\
13 \\
17 \\
\text { others } \\
\end{array}$ & $\begin{array}{l}16^{2}, 15^{6}, 8^{14}, 7^{2} \\
19,18^{2}, 15^{3}, 13^{3}, 9^{9}, 7,6^{2}, 3^{3} \\
19^{2}, 15^{5}, 11,10^{8}, 9,5^{7} \\
19^{2}, 17,15,14^{4}, 13,11^{3}, 9^{2}, 7^{7}, 3^{3} \\
11^{21}, 7^{2}, 3 \\
13^{15}, 9^{2}, 7^{3}, 5,3^{3} \\
17^{5}, 15,13^{3}, 11^{3}, 9^{3}, 7^{5}, 5,3^{3} \\
19^{2}, 17,15^{3}, 13^{3}, 11^{3}, 9^{3}, 7^{5}, 5,3^{3}\end{array}$ \\
\hline$E_{7}\left(a_{2}\right)$ & $\begin{array}{l}2 \\
3 \\
5 \\
13 \\
17 \\
19 \\
\text { others } \\
\end{array}$ & $\begin{array}{l}16^{4}, 14^{4}, 13^{4}, 12^{4}, 4^{2}, 3^{2}, 2^{6}, 1^{2} \\
19,18^{2}, 17^{3}, 10^{6}, 9^{7}, 8^{2}, 1^{3} \\
23,19,18^{2}, 17,16^{2}, 15^{2}, 11,10^{4}, 8^{2}, 7,4^{2}, 3^{2}, 1^{3} \\
13^{18}, 4^{2}, 3,1^{3} \\
17^{9}, 15,11,10^{2}, 9,8^{2}, 7,4^{2}, 3^{2}, 1^{3} \\
19^{3}, 18^{2}, 17,16^{2}, 15,11^{2}, 10^{2}, 9,8^{2}, 7,4^{2}, 3^{2}, 1^{3} \\
23,19,18^{2}, 17,16^{2}, 15^{2}, 11^{2}, 10^{2}, 9,8^{2}, 7,4^{2}, 3^{2}, 1^{3}\end{array}$ \\
\hline$E_{6}+A_{1}$ & $\begin{array}{l}2 \\
3 \\
13 \\
17 \\
19 \\
\text { others }\end{array}$ & $\begin{array}{l}16^{4}, 14^{4}, 13^{4}, 12^{4}, 4^{2}, 3^{2}, 2^{6}, 1^{2} \\
9^{25}, 8^{2}, 3,2^{2} \\
13^{18}, 3,2^{4}, 1^{3} \\
17^{9}, 15,10^{2}, 9^{3}, 8^{2}, 3^{2}, 2^{4}, 1^{3} \\
19^{2}, 18^{2}, 17^{3}, 16^{2}, 11,10^{2}, 9^{3}, 8^{2}, 3^{2}, 2^{4}, 1^{3} \\
23,18^{2}, 17^{3}, 16^{2}, 15,11,10^{2}, 9^{3}, 8^{2}, 3^{2}, 2^{4}, 1^{3}\end{array}$ \\
\hline$\left(D_{7}\left(a_{1}\right)\right)^{(2)}$ & 2 & $16^{2}, 15^{2}, 14^{4}, 9^{4}, 8^{8}, 6^{4}, 2^{2}, 1^{2}$ \\
\hline$D_{7}\left(a_{1}\right)$ & $\begin{array}{l}2 \\
3 \\
5 \\
11 \\
13 \\
17 \\
\text { others }\end{array}$ & $\begin{array}{l}8^{24}, 7^{8} \\
19,17^{2}, 15^{3}, 12^{2}, 11^{4}, 9^{5}, 6^{4}, 3^{4}, 1 \\
19,17^{2}, 15^{3}, 13,11^{3}, 10^{6}, 7^{2}, 5^{4}, 3^{3}, 1 \\
11^{20}, 9^{2}, 3^{3}, 1 \\
13^{13}, 11^{3}, 9,7^{2}, 5^{2}, 3^{4}, 1 \\
17^{4}, 15^{2}, 13,11^{6}, 9^{3}, 7^{3}, 5^{2}, 3^{4}, 1 \\
19,17^{2}, 15^{3}, 13,11^{6}, 9^{3}, 7^{3}, 5^{2}, 3^{4}, 1\end{array}$ \\
\hline$E_{8}\left(b_{6}\right)$ & $\begin{array}{l}2 \\
3 \\
5 \\
7 \\
11 \\
13 \\
\text { others } \\
\end{array}$ & $\begin{array}{l}16^{2}, 14^{2}, 13^{2}, 12^{2}, 11^{2}, 10^{2}, 9^{2}, 8^{4}, 6^{2}, 5^{2}, 4^{4}, 2^{4} \\
9^{25}, 8^{2}, 1^{7} \\
17,15^{3}, 13^{2}, 11^{3}, 10^{6}, 7^{3}, 5^{8}, 3^{2} \\
15,14^{6}, 11^{2}, 7^{17}, 5,3 \\
11^{18}, 9,7^{2}, 5^{3}, 3^{4} \\
13^{10}, 11^{3}, 9^{2}, 7^{5}, 5^{4}, 3^{4} \\
17,15^{3}, 13^{2}, 11^{6}, 9^{3}, 7^{5}, 5^{4}, 3^{4}\end{array}$ \\
\hline$E_{7}\left(a_{3}\right)$ & $\begin{array}{l}2 \\
3 \\
5 \\
11 \\
13 \\
17 \\
\text { others }\end{array}$ & $\begin{array}{l}16^{2}, 15^{2}, 14^{4}, 9^{4}, 8^{8}, 6^{4}, 2^{2}, 1^{2} \\
19,17,16^{2}, 15^{2}, 12^{2}, 11^{2}, 10^{4}, 9^{3}, 6^{4}, 3^{2}, 2^{2}, 1^{3} \\
19,17,16^{2}, 15^{2}, 12^{2}, 11^{2}, 10^{6}, 7,6^{2}, 5^{3}, 3,2^{2}, 1^{3} \\
11^{19}, 10^{2}, 9,3,2^{2}, 1^{3} \\
13^{12}, 12^{2}, 11,10^{2}, 7,6^{2}, 5,3^{2}, 2^{2}, 1^{3} \\
17^{3}, 16^{2}, 15,12^{2}, 11^{3}, 10^{4}, 9,7^{2}, 6^{2}, 5,3^{2}, 2^{2}, 1^{3} \\
19,17,16^{2}, 15^{2}, 12^{2}, 11^{3}, 10^{4}, 9,7^{2}, 6^{2}, 5,3^{2}, 2^{2}, 1^{3}\end{array}$ \\
\hline$E_{6}\left(a_{1}\right)+A_{1}$ & $\begin{array}{l}2 \\
3 \\
5 \\
7 \\
11 \\
13 \\
\text { others }\end{array}$ & $\begin{array}{l}16^{2}, 14^{2}, 13^{2}, 12^{2}, 11^{2}, 10^{2}, 9^{2}, 8^{4}, 6^{2}, 5^{2}, 4^{4}, 2^{4} \\
9^{25}, 8^{2}, 3,2^{2} \\
17,15,14^{2}, 13^{2}, 12^{2}, 11,10^{4}, 9^{2}, 8^{2}, 7,5^{7}, 3^{2}, 2^{2}, 1 \\
15,14^{4}, 13^{2}, 12^{2}, 7^{17}, 3,2^{2}, 1 \\
11^{18}, 9,6^{2}, 5^{2}, 4^{2}, 3^{2}, 2^{2}, 1 \\
13^{10}, 11,10^{2}, 9^{2}, 8^{2}, 7,6^{2}, 5^{3}, 4^{2}, 3^{2}, 2^{2}, 1 \\
17,15,14^{2}, 13^{2}, 12^{2}, 11^{2}, 10^{2}, 9^{3}, 8^{2}, 7,6^{2}, 5^{3}, 4^{2}, 3^{2}, 2^{2}, 1\end{array}$ \\
\hline$A_{7}^{(3)}$ & 3 & $9^{25}, 8^{2}, 3^{1}, 2^{2}$ \\
\hline$A_{7}$ & $\begin{array}{l}2 \\
3 \\
5 \\
7 \\
11 \\
13 \\
\text { others }\end{array}$ & $\begin{array}{l}8^{24}, 7^{8} \\
9^{25}, 8^{2}, 1^{7} \\
16^{2}, 15,13^{3}, 12^{2}, 11,10^{2}, 9^{3}, 8^{4}, 7,5^{7}, 3,1^{3} \\
15,14^{4}, 13^{3}, 8^{4}, 7^{13}, 6^{2}, 1^{3} \\
11^{17}, 8^{4}, 5^{3}, 4^{2}, 3,1^{3} \\
13^{9}, 12^{2}, 9^{3}, 8^{4}, 7,6^{2}, 5^{3}, 4^{2}, 3,1^{3} \\
16^{2}, 15,13^{3}, 12^{2}, 11,10^{2}, 9^{3}, 8^{4}, 7,6^{2}, 5^{3}, 4^{2}, 3,1^{3}\end{array}$ \\
\hline$D_{7}\left(a_{2}\right)$ & $\begin{array}{l}2 \\
3 \\
5 \\
7 \\
11 \\
13 \\
\text { others }\end{array}$ & $\begin{array}{l}8^{24}, 7^{8} \\
9^{23}, 7,5^{6}, 3,1 \\
15,14^{2}, 11,10^{10}, 9^{2}, 5^{15}, 1 \\
15,14^{2}, 13,12^{2}, 11^{2}, 10^{2}, 9^{2}, 8^{2}, 7^{9}, 5^{2}, 4^{2}, 3^{2}, 2^{2}, 1 \\
11^{14}, 9^{2}, 8^{2}, 7^{2}, 6^{2}, 5^{3}, 4^{2}, 3^{2}, 2^{2}, 1 \\
13^{7}, 11,10^{2}, 9^{3}, 8^{4}, 7^{3}, 6^{2}, 5^{3}, 4^{2}, 3^{2}, 2^{2}, 1 \\
15,14^{2}, 13,12^{2}, 11^{2}, 10^{2}, 9^{3}, 8^{4}, 7^{3}, 6^{2}, 5^{3}, 4^{2}, 3^{2}, 2^{2}, 1\end{array}$ \\
\hline
\end{tabular}


TABLE A.7. Jordan blocks of nilpotent elements on the adjoint module for $E_{8}$, III.

\begin{tabular}{|c|c|c|}
\hline$e$ & $p$ & Jordan blocks \\
\hline \multirow[t]{6}{*}{$E_{6}$} & 2 & $16^{4}, 13^{12}, 4^{2}, 3^{2}, 1^{14}$ \\
\hline & 3 & $9^{25}, 8^{2}, 1^{7}$ \\
\hline & 13 & $13^{18}, 1^{14}$ \\
\hline & 17 & $17^{9}, 15,9^{7}, 3,1^{14}$ \\
\hline & 19 & $19^{2}, 17^{7}, 11,9^{7}, 3,1^{14}$ \\
\hline & others & $23,17^{7}, 15,11,9^{7}, 3,1^{14}$ \\
\hline \multirow[t]{7}{*}{$D_{6}$} & 2 & $8^{22}, 7^{2}, 6^{8}, 2^{2}, 1^{6}$ \\
\hline & 3 & $19,16^{4}, 15,11^{5}, 10^{4}, 9^{2}, 6^{4}, 3,1^{10}$ \\
\hline & 5 & $19,16^{4}, 15,11^{6}, 10^{4}, 6^{4}, 5^{2}, 1^{10}$ \\
\hline & 11 & $11^{18}, 10^{4}, 1^{10}$ \\
\hline & 13 & $13^{12}, 11^{5}, 6^{4}, 3,1^{10}$ \\
\hline & & $17^{2}, 16^{4}, 11^{6}, 10^{4}, 7,6^{4}, 3,1^{10}$ \\
\hline & others & $19,16^{4}, 15,11^{6}, 10^{4}, 7,6^{4}, 3,1^{10}$ \\
\hline$\left(D_{5}+A_{2}\right)^{(2)}$ & 2 & $8^{22}, 7^{2}, 6^{8}, 2^{2}, 1^{6}$ \\
\hline \multirow{6}{*}{$D_{5}+A_{2}$} & 2 & $8^{20}, 7^{4}, 4^{12}, 3^{4}$ \\
\hline & 3 & $9^{19}, 8^{4}, 6^{4}, 3^{7}$ \\
\hline & 5 & $15,13^{2}, 11^{3}, 10^{8}, 9,7^{2}, 5^{11}, 3^{5}, 1$ \\
\hline & 11 & $11^{13}, 9^{3}, 7^{4}, 5^{5}, 3^{8}, 1$ \\
\hline & & $13^{4}, 11^{6}, 9^{5}, 7^{5}, 5^{5}, 3^{8}, 1$ \\
\hline & others & $15,13^{2}, 11^{7}, 9^{5}, 7^{5}, 5^{5}, 3^{8}, 1$ \\
\hline \multirow[t]{7}{*}{$E_{6}\left(a_{1}\right)$} & 2 & $16^{2}, 13^{6}, 11^{2}, 9^{6}, 8^{2}, 5^{6}, 4^{2}, 1^{8}$ \\
\hline & 3 & $9^{25}, 8^{2}, 1^{7}$ \\
\hline & 5 & $17,15,13^{6}, 11,10^{2}, 9^{6}, 7,5^{7}, 3,1^{8}$ \\
\hline & 7 & $15,14^{2}, 13^{6}, 7^{17}, 1^{8}$ \\
\hline & 11 & $11^{18}, 9,5^{6}, 3,1^{8}$ \\
\hline & & $13^{10}, 11,9^{6}, 7,5^{7}, 3,1^{8}$ \\
\hline & others & $17,15,13^{6}, 11^{2}, 9^{7}, 7,5^{7}, 3,1^{8}$ \\
\hline \multirow[t]{6}{*}{$E_{7}\left(a_{4}\right)$} & 2 & $8^{22}, 7^{2}, 6^{8}, 2^{2}, 1^{6}$ \\
\hline & 3 & $9^{19}, 8^{2}, 7^{4}, 6^{2}, 3^{6}, 1^{3}$ \\
\hline & 5 & $15,13,12^{2}, 11^{2}, 10^{8}, 8^{2}, 7,5^{10}, 4^{2}, 3^{2}, 2^{2}, 1^{3}$ \\
\hline & 11 & $11^{12}, 10^{2}, 9,8^{2}, 7^{2}, 6^{2}, 5^{2}, 4^{4}, 3^{4}, 2^{2}, 1^{3}$ \\
\hline & & $13^{3}, 12^{2}, 11^{3}, 10^{4}, 9^{2}, 8^{2}, 7^{3}, 6^{2}, 5^{2}, 4^{4}, 3^{4}, 2^{2}, 1^{3}$ \\
\hline & others & $15,13,12^{2}, 11^{4}, 10^{4}, 9^{2}, 8^{2}, 7^{3}, 6^{2}, 5^{2}, 4^{4}, 3^{4}, 2^{2}, 1^{3}$ \\
\hline \multirow[t]{5}{*}{$A_{6}+A_{1}$} & 2 & $8^{22}, 7^{2}, 6^{8}, 2^{2}, 1^{6}$ \\
\hline & 3 & $9^{19}, 8^{2}, 7^{4}, 6^{2}, 3^{6}, 1^{3}$ \\
\hline & 7 & $7^{35}, 3$ \\
\hline & & $11^{11}, 8^{4}, 7^{5}, 6^{4}, 5^{3}, 4^{2}, 3^{2}, 2^{2}, 1^{3}$ \\
\hline & others & $13^{3}, 12^{2}, 11,10^{2}, 9^{3}, 8^{4}, 7^{5}, 6^{4}, 5^{3}, 4^{2}, 3^{2}, 2^{2}, 1^{3}$ \\
\hline \multirow[t]{6}{*}{$D_{6}\left(a_{1}\right)$} & 2 & $8^{22}, 7^{2}, 6^{8}, 2^{2}, 1^{6}$ \\
\hline & 3 & $9^{19}, 7^{8}, 3^{5}, 1^{6}$ \\
\hline & 5 & $15,12^{4}, 11,10^{6}, 9^{4}, 7,5^{10}, 3^{5}, 1^{6}$ \\
\hline & 11 & $11^{12}, 9^{5}, 7,6^{4}, 4^{4}, 3^{6}, 1^{6}$ \\
\hline & & $13^{2}, 12^{4}, 11,10^{4}, 9^{5}, 7^{2}, 6^{4}, 4^{4}, 3^{6}, 1^{6}$ \\
\hline & others & $15,12^{4}, 11^{2}, 10^{4}, 9^{5}, 7^{2}, 6^{4}, 4^{4}, 3^{6}, 1^{6}$ \\
\hline$\left(A_{6}\right)^{(2)}$ & 2 & $8^{22}, 7^{2}, 6^{8}, 2^{2}, 1^{6}$ \\
\hline \multirow[t]{5}{*}{$A_{6}$} & 2 & $8^{14}, 7^{18}, 1^{10}$ \\
\hline & 3 & $9^{19}, 7^{8}, 3^{5}, 1^{6}$ \\
\hline & 7 & $7^{35}, 1^{3}$ \\
\hline & & $11^{11}, 7^{13}, 5^{3}, 3^{5}, 1^{6}$ \\
\hline & others & $13^{3}, 11^{5}, 9^{3}, 7^{13}, 5^{3}, 3^{5}, 1^{6}$ \\
\hline \multirow[t]{5}{*}{$E_{8}\left(a_{7}\right)$} & 2 & $8^{20}, 7^{4}, 4^{12}, 3^{4}$ \\
\hline & 3 & $9^{14}, 7^{2}, 6^{8}, 5^{6}, 3^{10}$ \\
\hline & 5 & $11,10^{6}, 9^{3}, 7^{5}, 5^{20}, 3^{5}$ \\
\hline & 7 & $7^{30}, 5^{4}, 3^{6}$ \\
\hline & others & $11^{4}, 9^{6}, 7^{10}, 5^{10}, 3^{10}$ \\
\hline \multirow[t]{6}{*}{$D_{5}+A_{1}$} & 2 & $8^{20}, 6^{4}, 5^{4}, 4^{6}, 3^{2}, 2^{6}, 1^{2}$ \\
\hline & 3 & $9^{15}, 8^{8}, 7^{4}, 3,2^{6}, 1^{6}$ \\
\hline & 5 & $15,12^{2}, 11^{5}, 10^{4}, 9^{3}, 8^{2}, 5^{10}, 3,2^{6}, 1^{6}$ \\
\hline & 11 & $11^{11}, 10^{2}, 9^{3}, 8^{2}, 6^{2}, 5^{4}, 4^{2}, 3^{2}, 2^{6}, 1^{6}$ \\
\hline & 13 & $13^{2}, 12^{2}, 11^{4}, 10^{4}, 9^{3}, 8^{2}, 7,6^{2}, 5^{4}, 4^{2}, 3^{2}, 2^{6}, 1^{6}$ \\
\hline & others & $15,12^{2}, 11^{5}, 10^{4}, 9^{3}, 8^{2}, 7,6^{2}, 5^{4}, 4^{2}, 3^{2}, 2^{6}, 1^{6}$ \\
\hline \multirow[t]{5}{*}{$E_{7}\left(a_{5}\right)$} & 2 & $8^{20}, 6^{4}, 5^{4}, 4^{6}, 3^{2}, 2^{6}, 1^{2}$ \\
\hline & 3 & $9^{13}, 8^{2}, 7,6^{8}, 5^{3}, 4^{6}, 3^{6}, 1^{3}$ \\
\hline & 5 & $11,10^{6}, 9,8^{4}, 7^{2}, 6^{2}, 5^{18}, 4^{2}, 3^{3}, 1^{3}$ \\
\hline & 7 & $7^{29}, 6^{2}, 5,4^{4}, 3^{3}, 1^{3}$ \\
\hline & others & $11^{3}, 10^{2}, 9^{3}, 8^{4}, 7^{5}, 6^{6}, 5^{4}, 4^{6}, 3^{6}, 1^{3}$ \\
\hline \multirow{5}{*}{$E_{6}\left(a_{3}\right)+A_{1}$} & 2 & $8^{20}, 6^{4}, 5^{4}, 4^{6}, 3^{2}, 2^{6}, 1^{2}$ \\
\hline & 3 & $9^{9}, 8^{2}, 6^{16}, 3^{17}, 2^{2}$ \\
\hline & 5 & $11,10^{4}, 9^{3}, 8^{4}, 7^{3}, 6^{2}, 5^{17}, 3^{3}, 2^{4}, 1^{3}$ \\
\hline & 7 & $7^{28}, 6^{2}, 5^{3}, 4^{2}, 3^{2}, 2^{4}, 1^{3}$ \\
\hline & others & $11^{2}, 10^{2}, 9^{4}, 8^{4}, 7^{4}, 6^{6}, 5^{7}, 4^{4}, 3^{4}, 2^{4}, 1^{3}$ \\
\hline
\end{tabular}


TABLE A.8. Jordan blocks of nilpotent elements on the adjoint module for $E_{8}, I V$.

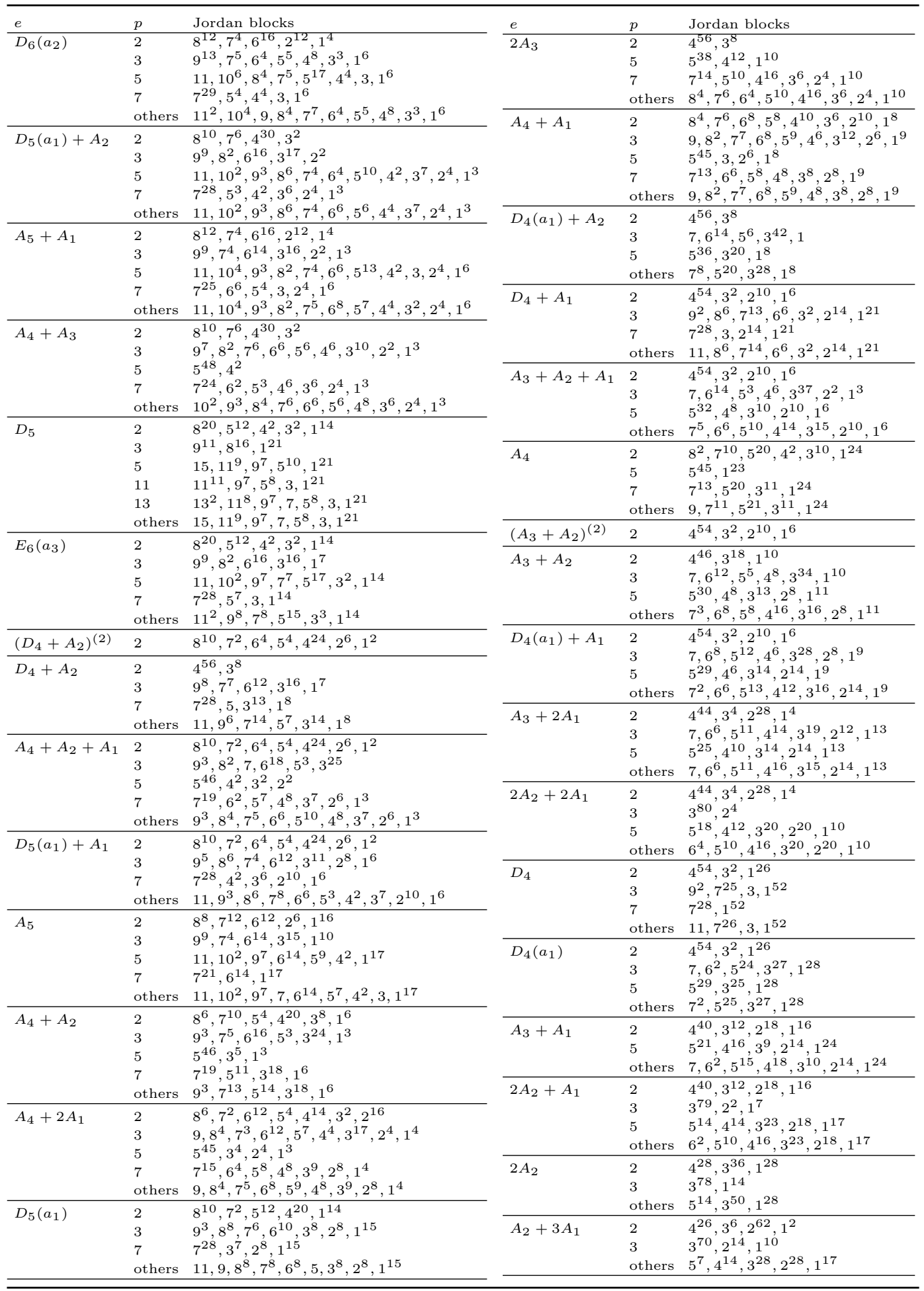


TABLE A.9. Jordan blocks of nilpotent elements on the adjoint module for $E_{8}, V$.

\begin{tabular}{lll}
\hline$e$ & $p$ & Jordan blocks \\
\hline$A_{3}$ & 2 & $4^{36}, 3^{20}, 1^{44}$ \\
& 5 & $5^{13}, 4^{32}, 1^{55}$ \\
& others & $7,5^{11}, 4^{32}, 3,1^{55}$ \\
\hline$A_{2}+2 A_{1}$ & 2 & $4^{22}, 3^{14}, 2^{52}, 1^{14}$ \\
& 3 & $3^{65}, 2^{16}, 1^{21}$ \\
& others & $5^{3}, 4^{16}, 3^{27}, 2^{32}, 1^{24}$ \\
\hline$A_{2}+A_{1}$ & 2 & $4^{14}, 3^{30}, 2^{34}, 1^{34}$ \\
& 3 & $3^{58}, 2^{20}, 1^{34}$ \\
\hline $4 A_{1}$ & others & $5,4^{12}, 3^{32}, 2^{32}, 1^{35}$ \\
\hline & 2 & $2^{120}, 1^{8}$ \\
& 3 & $3^{44}, 2^{40}, 1^{36}$ \\
\hline$A_{2}$ & others & $4^{8}, 3^{28}, 2^{48}, 1^{36}$ \\
& 2 & $4^{2}, 3^{54}, 1^{78}$ \\
& 3 & $3^{57}, 1^{77}$ \\
\hline $3 A_{1}$ & others & $5,3^{55}, 1^{78}$ \\
& 2 & $2^{110}, 1^{28}$ \\
& 3 & $3^{31}, 2^{50}, 1^{55}$ \\
\hline $2 A_{1}$ & others & $4^{2}, 3^{27}, 2^{52}, 1^{55}$ \\
\hline$A_{1}$ & 2 & $2^{92}, 1^{64}$ \\
& others & $3^{14}, 2^{64}, 1^{78}$ \\
\hline & 2 & $2^{58}, 1^{132}$ \\
& others & $3,2^{56}, 1^{133}$ \\
\hline & & \\
& &
\end{tabular}


TABLE A.10. Jordan blocks of nilpotent elements on the adjoint module for $E_{7}, I$.

\begin{tabular}{|c|c|c|c|c|c|}
\hline$e$ & $p$ & Jordan blocks & $e$ & $p$ & Jordan blocks \\
\hline \multirow[t]{11}{*}{$E_{7}$} & 2 & $16^{6}, 15^{2}, 2,1^{5}$ & \multirow[t]{6}{*}{$D_{6}\left(a_{1}\right)$} & 2 & $8^{14}, 7^{2}, 2,1^{5}$ \\
\hline & 3 & $27^{3}, 9^{4}, 8^{2}$ & & 3 & $9^{11}, 7^{4}, 3,1^{3}$ \\
\hline & 5 & $25^{4}, 15^{2}, 3$ & & 5 & $15,12^{2}, 11,10^{4}, 7,5^{6}, 3,1^{3}$ \\
\hline & 7 & $35,21^{4}, 7^{2}$ & & 11 & $11^{8}, 9,7,6^{2}, 4^{2}, 3^{2}, 1^{3}$ \\
\hline & 13 & $35,27,23,19,13^{2}, 3$ & & 13 & $13^{2}, 12^{2}, 11,10^{2}, 9,7^{2}, 6^{2}, 4^{2}, 3^{2}, 1^{3}$ \\
\hline & 17 & $35,27,23,17^{2}, 11,3$ & & others & $15,12^{2}, 11^{2}, 10^{2}, 9,7^{2}, 6^{2}, 4^{2}, 3^{2}, 1^{3}$ \\
\hline & 19 & $19^{7}$ & \multirow{6}{*}{$D_{5}+A_{1}$} & 2 & $8^{12}, 5^{4}, 4^{2}, 3^{2}, 2,1$ \\
\hline & 23 & $23^{5}, 15,3$ & & 3 & $9^{11}, 7^{4}, 3,1^{3}$ \\
\hline & 29 & $29^{2}, 27,19,15,11,3$ & & 5 & $15,12^{2}, 11,10^{2}, 9^{3}, 5^{6}, 3,1^{3}$ \\
\hline & & $31^{2}, 23,19,15,11,3$ & & 11 & $11^{7}, 9^{3}, 6^{2}, 4^{2}, 3^{2}, 1^{3}$ \\
\hline & others & $35,27,23,19,15,11,3$ & & & $13^{2}, 12^{2}, 10^{2}, 9^{3}, 7,6^{2}, 4^{2}, 3^{2}, 1^{3}$ \\
\hline \multirow[t]{9}{*}{$E_{7}\left(a_{1}\right)$} & 2 & $16^{6}, 15^{2}, 2,1^{5}$ & & others & $15,12^{2}, 11,10^{2}, 9^{3}, 7,6^{2}, 4^{2}, 3^{2}, 1^{3}$ \\
\hline & $\begin{array}{l}3 \\
5\end{array}$ & $27,19,17^{3}, 9^{4}$ & $\left(A_{6}\right)^{(2)}$ & 2 & $8^{14}, 7^{2}, 2,1^{5}$ \\
\hline & $\begin{array}{l}5 \\
7\end{array}$ & $\begin{array}{l}25^{2}, 19,17,15,11^{2}, 7,3 \\
27,21^{2}, 15,14^{2}, 7^{3}\end{array}$ & \multirow[t]{5}{*}{$A_{6}$} & 2 & $8^{14}, 7^{2}, 1^{7}$ \\
\hline & 11 & $23,22^{2}, 11^{6}$ & & 3 & $9^{11}, 7^{4}, 3,1^{3}$ \\
\hline & 13 & $27,23,19,17,13^{2}, 11,7,3$ & & 7 & $7^{19}$ \\
\hline & 17 & $17^{7}, 11,3$ & & 11 & $11^{7}, 7^{5}, 5^{3}, 3,1^{3}$ \\
\hline & 19 & $19^{5}, 17,11,7,3$ & & others & $13^{3}, 11,9^{3}, 7^{5}, 5^{3}, 3,1^{3}$ \\
\hline & 23 & $23^{3}, 17,15,11^{2}, 7,3$ & \multirow[t]{5}{*}{$E_{7}\left(a_{5}\right)$} & 2 & $8^{12}, 5^{4}, 4^{2}, 3^{2}, 2,1$ \\
\hline & others & $27,23,19,17,15,11^{2}, 7,3$ & & 3 & $9^{9}, 7,6^{2}, 5^{3}, 3^{6}$ \\
\hline \multirow[t]{7}{*}{$E_{7}\left(a_{2}\right)$} & 2 & $16^{4}, 13^{4}, 4^{2}, 3^{2}, 2,1$ & & 5 & $11,10^{4}, 9,7^{2}, 5^{10}, 3^{3}$ \\
\hline & 3 & $19,17^{3}, 9^{7}$ & & & $7^{17}, 5,3^{3}$ \\
\hline & 5 & $23,19,17,15^{2}, 11,10^{2}, 7,3^{2}$ & & others & $11^{3}, 9^{3}, 7^{5}, 5^{4}, 3^{6}$ \\
\hline & 13 & $13^{10}, 3$ & \multirow[t]{6}{*}{$D_{5}$} & 2 & $8^{12}, 5^{4}, 4^{2}, 3^{2}, 1^{3}$ \\
\hline & 17 & $17^{5}, 15,11,9,7,3^{2}$ & & 3 & $9^{7}, 8^{8}, 1^{6}$ \\
\hline & & $19^{3}, 17,15,11^{2}, 9,7,3^{2}$ & & 5 & $15,11^{5}, 9^{3}, 5^{6}, 1^{6}$ \\
\hline & others & $23,19,17,15^{2}, 11^{2}, 9,7,3^{2}$ & & 11 & $11^{7}, 9^{3}, 5^{4}, 3,1^{6}$ \\
\hline \multirow[t]{7}{*}{$E_{7}\left(a_{3}\right)$} & 2 & $16^{2}, 15^{2}, 9^{4}, 8^{4}, 2,1$ & & $\begin{array}{l}13 \\
\text { others }\end{array}$ & $13^{2}, 11^{4}, 9^{3}, 7,5^{4}, 3,1^{6}$ \\
\hline & 3 & $19,17,15^{2}, 11^{2}, 9^{3}, 6^{2}, 3^{2}$ & & others & $15,11^{5}, 9^{3}, 7,5^{4}, 3,1^{6}$ \\
\hline & 5 & $19,17,15^{2}, 11^{2}, 10^{2}, 7,5^{3}, 3$ & \multirow[t]{5}{*}{$E_{6}\left(a_{3}\right)$} & 2 & $8^{12}, 5^{4}, 4^{2}, 3^{2}, 1^{3}$ \\
\hline & 11 & $11^{11}, 9,3$ & & 3 & $9^{5}, 8^{2}, 6^{8}, 3^{8}$ \\
\hline & 13 & $13^{8}, 11,7,5,3^{2}$ & & 5 & $11,10^{2}, 9^{3}, 7^{3}, 5^{9}, 3^{2}, 1^{3}$ \\
\hline & & $17^{3}, 15,11^{3}, 9,7^{2}, 5,3^{2}$ & & 7 & $7^{16}, 5^{3}, 3,1^{3}$ \\
\hline & others & $19,17,15^{2}, 11^{3}, 9,7^{2}, 5,3^{2}$ & & others & $11^{2}, 9^{4}, 7^{4}, 5^{7}, 3^{3}, 1^{3}$ \\
\hline \multirow[t]{6}{*}{$E_{6}$} & 2 & $16^{4}, 13^{4}, 4^{2}, 3^{2}, 1^{3}$ & \multirow[t]{5}{*}{$D_{6}\left(a_{2}\right)$} & 2 & $8^{8}, 7^{4}, 6^{4}, 2^{7}, 1^{3}$ \\
\hline & 3 & $9^{13}, 8^{2}$ & & 3 & $9^{9}, 7,6^{2}, 5,4^{4}, 3^{3}, 1^{3}$ \\
\hline & 13 & $13^{10}, 1^{3}$ & & 5 & $11,10^{4}, 8^{2}, 7,5^{9}, 4^{2}, 3,1^{3}$ \\
\hline & 17 & $17^{5}, 15,9^{3}, 3,1^{3}$ & & 7 & $7^{17}, 4^{2}, 3,1^{3}$ \\
\hline & & $19^{2}, 17^{3}, 11,9^{3}, 3,1^{3}$ & & others & $11^{2}, 10^{2}, 9,8^{2}, 7^{3}, 6^{2}, 5,4^{4}, 3^{3}, 1^{3}$ \\
\hline & others & $23,17^{3}, 15,11,9^{3}, 3,1^{3}$ & \multirow[t]{4}{*}{$D_{5}\left(a_{1}\right)+A_{1}$} & 2 & $8^{6}, 7^{2}, 5^{4}, 4^{12}, 2,1$ \\
\hline \multirow{7}{*}{$E_{6}\left(a_{1}\right)$} & 2 & $16^{2}, 13^{2}, 11^{2}, 9^{2}, 8^{2}, 5^{2}, 4^{2}, 1$ & & 3 & $9^{5}, 7^{4}, 6^{6}, 3^{7}, 1^{3}$ \\
\hline & 3 & $9^{13}, 8^{2}$ & & 7 & $7^{16}, 3^{6}, 1^{3}$ \\
\hline & 5 & $17,15,13^{2}, 11,10^{2}, 9^{2}, 7,5^{3}, 3,1$ & & others & $11,9^{3}, 7^{8}, 5^{3}, 3^{7}, 1^{3}$ \\
\hline & 7 & $15,14^{2}, 13^{2}, 7^{9}, 1$ & \multirow{5}{*}{$A_{5}+A_{1}$} & 2 & $8^{8}, 7^{4}, 6^{4}, 2^{7}, 1^{3}$ \\
\hline & 11 & $11^{10}, 9,5^{2}, 3,1$ & & 3 & $9^{9}, 3^{16}, 2^{2}$ \\
\hline & & $13^{6}, 11,9^{2}, 7,5^{3}, 3,1$ & & 5 & $11,10^{2}, 9^{3}, 8^{2}, 5^{9}, 3,2^{4}, 1^{3}$ \\
\hline & others & $17,15,13^{2}, 11^{2}, 9^{3}, 7,5^{3}, 3,1$ & & & $7^{17}, 3,2^{4}, 1^{3}$ \\
\hline$D_{6}$ & 2 & $8^{14}, 7^{2}, 2,1^{5}$ & & others & $11,10^{2}, 9^{3}, 8^{2}, 7,6^{2}, 5^{3}, 4^{2}, 3^{2}, 2^{4}, 1^{3}$ \\
\hline & 3 & $19,16^{2}, 15,11,10^{2}, 9^{2}, 6^{2}, 3,1^{3}$ & $\left(A_{5}\right)^{\prime}$ & 2 & $8^{8}, 7^{4}, 6^{4}, 2^{6}, 1^{5}$ \\
\hline & 5 & $19,16^{2}, 15,11^{2}, 10^{2}, 6^{2}, 5^{2}, 1^{3}$ & & 3 & $9^{5}, 7^{4}, 6^{6}, 3^{7}, 1^{3}$ \\
\hline & 11 & $11^{10}, 10^{2}, 1^{3}$ & & 5 & $11,10^{2}, 9^{3}, 6^{6}, 5^{5}, 4^{2}, 1^{6}$ \\
\hline & 13 & $13^{8}, 11,6^{2}, 3,1^{3}$ & & 7 & $7^{13}, 6^{6}, 1^{6}$ \\
\hline & & $17^{2}, 16^{2}, 11^{2}, 10^{2}, 7,6^{2}, 3,1^{3}$ & & others & $11,10^{2}, 9^{3}, 7,6^{6}, 5^{3}, 4^{2}, 3,1^{6}$ \\
\hline & others & $19,16^{2}, 15,11^{2}, 10^{2}, 7,6^{2}, 3,1^{3}$ & $A_{4}+A_{2}$ & 2 & $8^{6}, 7^{2}, 5^{4}, 4^{12}, 1^{3}$ \\
\hline$E_{7}\left(a_{4}\right)$ & 2 & $8^{14}, 7^{2}, 2,1^{5}$ & & 3 & $9^{3}, 7,6^{8}, 5^{3}, 3^{12}$ \\
\hline & 3 & $9^{11}, 8^{2}, 6^{2}, 3^{2}$ & & 5 & $5^{26}, 3$ \\
\hline & 5 & $15,13,11^{2}, 10^{4}, 7,5^{6}, 3^{2}$ & & & $7^{11}, 5^{7}, 3^{6}, 1^{3}$ \\
\hline & 11 & $11^{8}, 9,7^{2}, 5^{2}, 3^{4}$ & & others & $9^{3}, 7^{5}, 5^{10}, 3^{6}, 1^{3}$ \\
\hline & & $13^{3}, 11^{3}, 9^{2}, 7^{3}, 5^{2}, 3^{4}$ & $D_{5}\left(a_{1}\right)$ & 2 & $8^{6}, 7^{2}, 5^{4}, 4^{12}, 1^{3}$ \\
\hline & others & $15,13,11^{4}, 9^{2}, 7^{3}, 5^{2}, 3^{4}$ & $50\left(\omega_{1}\right)$ & 3 & $9^{3}, 8^{4}, 7^{2}, 6^{6}, 3^{4}, 2^{4}, 1^{4}$ \\
\hline & & & & 7 & $7^{16}, 3^{3}, 2^{4}, 1^{4}$ \\
\hline & & & & others & $11,9,8^{4}, 7^{4}, 6^{4}, 5,3^{4}, 2^{4}, 1^{4}$ \\
\hline
\end{tabular}


TABLE A.11. Jordan blocks of nilpotent elements on the adjoint module for $E_{7}$, II.

\begin{tabular}{|c|c|c|c|c|c|}
\hline$e$ & $p$ & Jordan blocks & $e$ & $p$ & Jordan blocks \\
\hline \multirow[t]{4}{*}{$A_{4}+A_{1}$} & 2 & $8^{4}, 7^{2}, 6^{4}, 5^{4}, 4^{6}, 3^{2}, 2^{6}, 1$ & \multirow{3}{*}{$\left(A_{3}+A_{1}\right)^{\prime}$} & 2 & $4^{24}, 3^{4}, 2^{10}, 1^{5}$ \\
\hline & 3 & $9,8^{2}, 7^{3}, 6^{4}, 5^{5}, 4^{2}, 3^{8}, 2^{2}, 1^{2}$ & & & $5^{13}, 4^{8}, 3^{5}, 2^{6}, 1^{9}$ \\
\hline & & $5^{25}, 3,2^{2}, 1$ & & others & $7,6^{2}, 5^{7}, 4^{10}, 3^{6}, 2^{6}, 1^{9}$ \\
\hline & $\begin{array}{l}7 \\
\text { others }\end{array}$ & $\begin{array}{l}7^{9}, 6^{2}, 5^{4}, 4^{4}, 3^{4}, 2^{4}, 1^{2} \\
9,8^{2}, 7^{3}, 6^{4}, 5^{5}, 4^{4}, 3^{4}, 2^{4}, 1^{2}\end{array}$ & \multirow[t]{3}{*}{$2 A_{2}+A_{1}$} & & $\begin{array}{l}4^{24}, 3^{4}, 2^{10}, 1^{5} \\
3^{43}, 2^{2}\end{array}$ \\
\hline \multirow[t]{3}{*}{$D_{4}+A_{1}$} & \multirow{3}{*}{$\begin{array}{l}2 \\
3 \\
7 \\
\text { others }\end{array}$} & \multirow{3}{*}{$\begin{array}{l}4^{30}, 3^{2}, 2,1^{5} \\
9^{2}, 8^{4}, 7^{5}, 6^{4}, 3^{2}, 2^{4}, 1^{10} \\
7^{16}, 3,2^{4}, 1^{10} \\
11,8^{4}, 7^{6}, 6^{4}, 3^{2}, 2^{4}, 1^{10}\end{array}$} & & & $5^{10}, 4^{6}, 3^{11}, 2^{10}, 1^{6}$ \\
\hline & & & & others & $6^{2}, 5^{6}, 4^{8}, 3^{11}, 2^{10}, 1^{6}$ \\
\hline & & & $\left(A_{3}+A_{1}\right)^{\prime \prime}$ & & $\begin{array}{l}4^{20}, 3^{12}, 2,1^{15} \\
5^{17}, 3^{9}, 1^{21}\end{array}$ \\
\hline \multirow[t]{5}{*}{$\left(A_{5}\right)^{\prime \prime}$} & \multirow{5}{*}{$\begin{array}{l}2 \\
3 \\
5 \\
7 \\
\text { others } \\
\end{array}$} & \multirow{5}{*}{$\begin{array}{l}8^{4}, 7^{12}, 2,1^{15} \\
9^{9}, 3^{15}, 1^{7} \\
11,9^{7}, 5^{9}, 1^{14} \\
7^{17}, 1^{14} \\
11,9^{7}, 7,5^{7}, 3,1^{14}\end{array}$} & & others & $7,5^{15}, 3^{10}, 1^{21}$ \\
\hline & & & $A_{2}+3 A_{1}$ & 2 & $4^{14}, 3^{6}, 2^{29}, 1$ \\
\hline & & & $1_{2}+T_{1}$ & & $3^{42}, 1^{7}$ \\
\hline & & & & others & $5^{7}, 3^{28}, 1^{14}$ \\
\hline & & & $2 A_{2}$ & & $4^{20}, 3^{12}, 1^{17}$ \\
\hline \multirow[t]{3}{*}{$A_{3}+A_{2}+A_{1}$} & \multirow{3}{*}{$\begin{array}{l}2 \\
3 \\
5 \\
\text { others }\end{array}$} & \multirow{3}{*}{$\begin{array}{l}4^{30}, 3^{2}, 2,1^{5} \\
7,6^{8}, 5^{3}, 3^{21} \\
5^{20}, 3^{10}, 1^{3} \\
7^{5}, 5^{10}, 3^{15}, 1^{3}\end{array}$} & $2 \pi_{2}$ & & $3^{42}, 1^{7}$ \\
\hline & & & & others & $5^{10}, 3^{22}, 1^{17}$ \\
\hline & & & $A_{3}$ & & $4^{20}, 3^{12}, 1^{17}$ \\
\hline \multirow[t]{3}{*}{$A_{4}$} & \multirow{3}{*}{$\begin{array}{l}2 \\
5 \\
7 \\
\text { others }\end{array}$} & \multirow{3}{*}{$\begin{array}{l}8^{2}, 7^{6}, 5^{8}, 4^{2}, 3^{6}, 1^{9} \\
5^{25}, 1^{8} \\
7^{9}, 5^{8}, 3^{7}, 1^{9} \\
9,7^{7}, 5^{9}, 3^{7}, 1^{9}\end{array}$} & & others & $7,5^{7}, 4^{16}, 3,1^{24}$ \\
\hline & & & $A_{2}+2 A_{1}$ & 2 & $4^{14}, 3^{6}, 2^{28}, 1^{3}$ \\
\hline & & & & & $\begin{array}{l}3^{37}, 2^{8}, 1^{6} \\
5^{3} 4^{8}, 3^{15} 2^{16} 1^{9}\end{array}$ \\
\hline$\left(A_{3}+A_{2}\right)^{(2)}$ & 2 & $4^{30}, 3^{2}, 2,1^{5}$ & $A_{2}+A_{1}$ & & $4^{10} \cdot 3^{14} \cdot 2^{18} \cdot 1^{15}$ \\
\hline \multirow{3}{*}{$A_{3}+A_{2}$} & \multirow{3}{*}{$\begin{array}{l}2 \\
3 \\
5 \\
\text { others }\end{array}$} & \multirow{3}{*}{$\begin{array}{l}4^{30}, 3^{2}, 1^{7} \\
7,6^{8}, 5,4^{4}, 3^{18}, 1^{3} \\
5^{18}, 4^{4}, 3^{5}, 2^{4}, 1^{4} \\
7^{3}, 6^{4}, 5^{4}, 4^{8}, 3^{8}, 2^{4}, 1^{4}\end{array}$} & & & $3^{34}, 2^{8}, 1^{15}$ \\
\hline & & & & others & $5,4^{8}, 3^{16}, 2^{16}, 1^{16}$ \\
\hline & & & $4 A_{1}$ & & $2^{63}, 1^{7}$ \\
\hline \multirow[t]{4}{*}{$D_{4}\left(a_{1}\right)+A_{1}$} & \multirow{4}{*}{$\begin{array}{l}2 \\
3 \\
5 \\
\text { others }\end{array}$} & \multirow{4}{*}{$\begin{array}{l}4^{30}, 3^{2}, 2,1^{5} \\
7,6^{6}, 5^{4}, 4^{4}, 3^{16}, 1^{6} \\
5^{17}, 4^{4}, 3^{6}, 2^{4}, 1^{6} \\
7^{2}, 6^{4}, 5^{5}, 4^{8}, 3^{8}, 2^{4}, 1^{6}\end{array}$} & & $\begin{array}{l}3 \\
\text { others }\end{array}$ & $\begin{array}{l}3^{20}, 2^{16}, 1^{20} \\
4^{6}, 3^{16}, 2^{20}, 1^{21}\end{array}$ \\
\hline & & & $A_{2}$ & 2 & $4^{2}, 3^{30}, 1^{35}$ \\
\hline & & & $A_{2}$ & $\begin{array}{l}2 \\
3\end{array}$ & $3^{33}, 1^{34}$ \\
\hline & & & & others & $5,3^{31}, 1^{35}$ \\
\hline \multirow[t]{4}{*}{$D_{4}$} & 2 & $4^{30}, 3^{2}, 1^{7}$ & \multirow{3}{*}{$\left(3 A_{1}\right)^{\prime}$} & 2 & $2^{62}, 1^{9}$ \\
\hline & 3 & $9^{2}, 7^{13}, 3,1^{21}$ & & & $3^{19}, 2^{26}, 1^{24}$ \\
\hline & & $7^{16}, 1^{21}$ & & others & $4^{2}, 3^{15}, 2^{28}, 1^{24}$ \\
\hline & others & $11,7^{14}, 3,1^{21}$ & \multirow{2}{*}{$\left(3 A_{1}\right)^{\prime \prime}$} & & $2^{53}, 1^{27}$ \\
\hline \multirow[t]{3}{*}{$A_{3}+2 A_{1}$} & \multirow{3}{*}{$\begin{array}{l}2 \\
3 \\
5 \\
\text { others }\end{array}$} & \multirow{3}{*}{$\begin{array}{l}4^{24}, 3^{4}, 2^{11}, 1^{3} \\
7,6^{4}, 5^{7}, 4^{4}, 3^{11}, 2^{6}, 1^{6} \\
5^{17}, 4^{2}, 3^{6}, 2^{8}, 1^{6} \\
7,6^{4}, 5^{7}, 4^{6}, 3^{7}, 2^{8}, 1^{6}\end{array}$} & & others & $3^{27}, 1^{52}$ \\
\hline & & & $2 A_{1}$ & & $2^{52}, 1^{29}$ \\
\hline & & & & others & $3^{10}, 2^{32}, 1^{39}$ \\
\hline \multirow[t]{3}{*}{$D_{4}\left(a_{1}\right)$} & 2 & $4^{30}, 3^{2}, 1^{7}$ & $A_{1}$ & $\begin{array}{l}2 \\
\text { others }\end{array}$ & $\begin{array}{l}2^{34}, 1^{65} \\
3,2^{32}, 1^{66}\end{array}$ \\
\hline & $\begin{array}{l}3 \\
5\end{array}$ & $\begin{array}{l}7,6^{2}, 5^{12}, 3^{15}, 1^{9} \\
5^{17}, 3^{13}, 1^{9}\end{array}$ & & & \\
\hline & others & $7^{2}, 5^{13}, 3^{15}, 1^{9}$ & & & \\
\hline
\end{tabular}


TABLE A.12. Jordan blocks of nilpotent elements on the adjoint module for $E_{6}$.

\begin{tabular}{|c|c|c|c|c|c|}
\hline$e$ & $p$ & Jordan blocks & $e$ & $p$ & Jordan blocks \\
\hline \multirow[t]{6}{*}{$E_{6}$} & 2 & $16^{4}, 4^{2}, 3^{2}$ & \multirow[t]{4}{*}{$A_{4}$} & 2 & $8^{2}, 7^{4}, 5^{2}, 4^{2}, 3^{4}, 1^{4}$ \\
\hline & 3 & $9^{6}, 8^{3}$ & & 5 & $5^{15}, 1^{3}$ \\
\hline & 13 & $13^{6}$ & & 7 & $7^{7}, 5^{2}, 3^{5}, 1^{4}$ \\
\hline & 17 & $17^{3}, 15,9,3$ & & others & $9,7^{5}, 5^{3}, 3^{5}, 1^{4}$ \\
\hline & & $19^{2}, 17,11,9,3$ & \multirow[t]{4}{*}{$D_{4}\left(a_{1}\right)$} & 2 & $4^{18}, 3^{2}$ \\
\hline & others & $23,17,15,11,9,3$ & & 3 & $7,6^{2}, 5^{6}, 3^{9}, 1^{2}$ \\
\hline \multirow[t]{6}{*}{$E_{6}\left(a_{1}\right)$} & 2 & $16^{2}, 11^{2}, 8^{2}, 4^{2}$ & & 5 & $5^{11}, 3^{7}, 1^{2}$ \\
\hline & 3 & $9^{6}, 8^{3}$ & & others & $7^{2}, 5^{7}, 3^{9}, 1^{2}$ \\
\hline & \multirow{4}{*}{$\begin{array}{l}5 \\
7 \\
11 \\
13 \\
\text { others } \\
\end{array}$} & \multirow{4}{*}{$\begin{array}{l}17,15,11,10^{2}, 7,5,3 \\
15,14^{2}, 7^{5} \\
11^{6}, 9,3 \\
13^{4}, 11,7,5,3 \\
17,15,11^{2}, 9,7,5,3\end{array}$} & \multirow{3}{*}{$A_{3}+A_{1}$} & 2 & $4^{16} \cdot 2^{6} \cdot 1^{2}$ \\
\hline & & & & & $5^{9}, 4^{4}, 3^{3}, 2^{2}, 1^{4}$ \\
\hline & & & & others & $7,6^{2}, 5^{3}, 4^{6}, 3^{4}, 2^{2}, 1^{4}$ \\
\hline & & & $2 A_{2}+A_{1}$ & 2 & $4^{16}, 2^{6}, 1^{2}$ \\
\hline \multirow[t]{5}{*}{$D_{5}$} & \multirow{5}{*}{$\begin{array}{l}2 \\
3 \\
5 \\
11 \\
13 \\
\text { others } \\
\end{array}$} & \multirow{5}{*}{$\begin{array}{l}8^{8}, 4^{2}, 3^{2} \\
9^{5}, 8^{4}, 1 \\
15,11^{3}, 9,5^{4}, 1 \\
11^{5}, 9,5^{2}, 3,1 \\
13^{2}, 11^{2}, 9,7,5^{2}, 3,1 \\
15,11^{3}, 9,7,5^{2}, 3,1 \\
\end{array}$} & & & 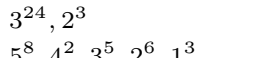 \\
\hline & & & & $\begin{array}{l}5 \\
\text { others }\end{array}$ & $6^{2}, 5^{4}, 4^{4}, 3^{5}, 2^{6}, 1^{3}$ \\
\hline & & & $A_{3}$ & 2 & $4^{12}, 3^{8}, 1^{6}$ \\
\hline & & & & 5 & $5^{7}, 4^{8}, 1^{11}$ \\
\hline & & & & others & $7,5^{5}, 4^{8}, 3,1^{11}$ \\
\hline \multirow{5}{*}{$E_{6}\left(a_{3}\right)$} & 2 & $8^{8}, 4^{2}, 3^{2}$ & \multirow[t]{3}{*}{$A_{2}+2 A_{1}$} & 2 & $4^{10}, 3^{2}, 2^{16}$ \\
\hline & 3 & $9^{3}, 8^{2}, 6^{4}, 3^{3}, 2$ & & 3 & $3^{23}, 2^{4}, 1$ \\
\hline & 5 & $11,10^{2}, 9,7,5^{5}, 3^{2}$ & & others & $5^{3}, 4^{4}, 3^{9}, 2^{8}, 1^{4}$ \\
\hline & 7 & $7^{10}, 5,3$ & \multirow{3}{*}{$2 A_{2}$} & 2 & $4^{16}, 1^{14}$ \\
\hline & others & $11^{2}, 9^{2}, 7^{2}, 5^{3}, 3^{3}$ & & & $3^{23}, 2,1^{7}$ \\
\hline \multirow[t]{4}{*}{$D_{5}\left(a_{1}\right)$} & 2 & $8^{4}, 7^{2}, 4^{8}$ & & others & $5^{8}, 3^{8}, 1^{14}$ \\
\hline & 3 & $9^{3}, 8^{2}, 6^{4}, 3^{2}, 2^{2}, 1$ & \multirow[t]{3}{*}{$A_{2}+A_{1}$} & 2 & $4^{8}, 3^{6}, 2^{10}, 1^{8}$ \\
\hline & 7 & $7^{10}, 3,2^{2}, 1$ & & & $3^{22}, 2^{2}, 1^{8}$ \\
\hline & others & $11,9,8^{2}, 7^{2}, 6^{2}, 5,3^{2}, 2^{2}, 1$ & & others & $5,4^{6}, 3^{8}, 2^{8}, 1^{9}$ \\
\hline \multirow[t]{5}{*}{$A_{5}$} & 2 & $8^{8}, 2^{6}, 1^{2}$ & \multirow[t]{3}{*}{$A_{2}$} & 2 & $4^{2}, 3^{18}, 1^{16}$ \\
\hline & 3 & $9^{3}, 7^{4}, 6^{2}, 3^{2}, 2,1^{3}$ & & 3 & $3^{21}, 1^{15}$ \\
\hline & 5 & $11,10^{2}, 9,6^{2}, 5^{3}, 4^{2}, 1^{3}$ & & others & $5,3^{19}, 1^{16}$ \\
\hline & 7 & $7^{9}, 6^{2}, 1^{3}$ & \multirow[t]{3}{*}{$3 A_{1}$} & 2 & $2^{38}, 1^{2}$ \\
\hline & others & $11,10^{2}, 9,7,6^{2}, 5,4^{2}, 3,1^{3}$ & & & $3^{13}, 2^{14}, 1^{11}$ \\
\hline \multirow[t]{4}{*}{$A_{4}+A_{1}$} & 2 & $8^{4}, 6^{2}, 5^{2}, 4^{4}, 2^{4}$ & & others & $4^{2}, 3^{9}, 2^{16}, 1^{11}$ \\
\hline & 3 & $9,8^{2}, 7,6^{2}, 5^{3}, 3^{6}, 1$ & \multirow{2}{*}{$2 A_{1}$} & 2 & $2^{32}, 1^{14}$ \\
\hline & $\begin{array}{l}5 \\
7\end{array}$ & $\begin{array}{l}5^{15}, 3 \\
7^{7}, 5^{2} 4^{2} 3^{2} 2^{2}\end{array}$ & & others & $3^{8}, 2^{16}, 1^{22}$ \\
\hline & others & $9,8^{2}, 7,6^{2}, 5^{3}, 4^{2}, 3^{2}, 2^{2}, 1$ & \multirow[t]{2}{*}{$A_{1}$} & 2 & $2^{22}, 1^{34}$ \\
\hline \multirow[t]{4}{*}{$D_{4}$} & 2 & $4^{18}, 3^{2}$ & & others & $3,2^{20}, 1^{35}$ \\
\hline & 3 & $9^{2}, 7^{7}, 3,1^{8}$ & & & \\
\hline & 7 & $7^{10}, 1^{8}$ & & & \\
\hline & others & $11,7^{8}, 3,1^{8}$ & & & \\
\hline
\end{tabular}


TABLE A.13. Jordan blocks of nilpotent elements on the adjoint modules for $F_{4}$ and $G_{2}$.

\begin{tabular}{|c|c|c|c|c|c|}
\hline$e$ & $p$ & Jordan blocks & $e$ & $p$ & Jordan blocks \\
\hline \multirow[t]{6}{*}{$F_{4}$} & 2 & $16^{2}, 4^{2}, 3^{4}$ & \multirow[t]{5}{*}{$G_{2}$} & 2 & $4^{2}, 3^{2}$ \\
\hline & 3 & $9^{4}, 8^{2}$ & & 2 & $4^{2}, 3^{2}$ \\
\hline & 13 & $13^{4}$ & & 3 & $9,3,2$ \\
\hline & 17 & $17^{2}, 15,3$ & & 7 & \\
\hline & \multirow{2}{*}{$\begin{array}{l}19 \\
\text { others }\end{array}$} & $19^{2}, 11,3$ & & others & 11,3 \\
\hline & & $23,15,11,3$ & \multirow[t]{4}{*}{$G_{2}\left(a_{1}\right)$} & 2 & $4^{2}, 3^{2}$ \\
\hline \multirow[t]{5}{*}{$F_{4}\left(a_{1}\right)$} & \multirow{5}{*}{$\begin{array}{l}2 \\
3 \\
5 \\
11 \\
13 \\
\text { others } \\
\end{array}$} & \multirow{5}{*}{$\begin{array}{l}8^{4}, 4^{2}, 3^{4} \\
9^{4}, 8^{2} \\
15,11^{2}, 5^{3} \\
11^{4}, 5,3 \\
13^{2}, 11,7,5,3 \\
15,11^{2}, 7,5,3\end{array}$} & & 2 & $4^{2}, 3^{2}$ \\
\hline & & & & 3 & $3^{4}, 2$ \\
\hline & & & & others & $5,3^{3}$ \\
\hline & & & $\left(\tilde{A}_{1}\right)^{(3)}$ & 3 & $3^{3}, 2^{2}, 1$ \\
\hline & & & $\tilde{A}_{1}$ & & $2^{6}, 1^{2}$ \\
\hline \multirow[t]{4}{*}{$F_{4}\left(a_{2}\right)$} & \multirow{4}{*}{$\begin{array}{l}2 \\
3 \\
5 \\
7 \\
\text { others } \\
\end{array}$} & \multirow{4}{*}{$\begin{array}{l}8^{4}, 4^{2}, 3^{4} \\
9^{2}, 8^{2}, 6^{2}, 3^{2} \\
11,10^{2}, 5^{3}, 3^{2} \\
7^{7}, 3 \\
11^{2}, 9,7,5,3^{3}\end{array}$} & & & $2^{6}, 1^{2}$ \\
\hline & & & & others & $4^{2}, 3,1^{3}$ \\
\hline & & & $A_{1}$ & & $2^{6}, 1^{2}$ \\
\hline & & & & & $2^{6}, 1^{2}$ \\
\hline$\left(C_{3}\right)^{(2)}$ & 2 & $8^{4}, 4,3^{3}, 2^{3}, 1$ & & $\begin{array}{l}3 \\
\text { others }\end{array}$ & $\begin{array}{l}3,2^{4}, 1^{3} \\
4^{2}, 3,1^{3}\end{array}$ \\
\hline \multirow[t]{5}{*}{$C_{3}$} & 2 & $8^{4}, 2^{8}, 1^{4}$ & & & \\
\hline & 3 & $9^{2}, 7^{4}, 3,1^{3}$ & & & \\
\hline & 5 & $11,10^{2}, 5^{2}, 4^{2}, 1^{3}$ & & & \\
\hline & & $7^{7}, 1^{3}$ & & & \\
\hline & others & $11,10^{2}, 7,4^{2}, 3,1^{3}$ & & & \\
\hline \multirow[t]{4}{*}{$B_{3}$} & 2 & $4^{10}, 3^{4}$ & & & \\
\hline & 3 & $9^{2}, 7^{4}, 3,1^{3}$ & & & \\
\hline & 7 & $7^{7}, 1^{3}$ & & & \\
\hline & others & $11,7^{5}, 3,1^{3}$ & & & \\
\hline \multirow[t]{4}{*}{$F_{4}\left(a_{3}\right)$} & 2 & $4^{10}, 3^{4}$ & & & \\
\hline & 3 & $7,6^{2}, 5^{3}, 3^{6}$ & & & \\
\hline & & $5^{8}, 3^{4}$ & & & \\
\hline & others & $7^{2}, 5^{4}, 3^{6}$ & & & \\
\hline$C_{3}\left(a_{1}\right)^{(2)}$ & 2 & $4^{9}, 3^{3}, 2^{3}, 1$ & & & \\
\hline \multirow{3}{*}{$C_{3}\left(a_{1}\right)$} & 2 & $4^{8}, 2^{8}, 1^{4}$ & & & \\
\hline & & $5^{7}, 4^{2}, 3^{2}, 1^{3}$ & & & \\
\hline & others & $7,6^{2}, 5,4^{4}, 3^{3}, 1^{3}$ & & & \\
\hline$\left(\tilde{A}_{2}+A_{1}\right)^{(2)}$ & 2 & $4^{9}, 3^{3}, 2^{3}, 1$ & & & \\
\hline \multirow[t]{4}{*}{$\tilde{A}_{2}+A_{1}$} & 2 & $4^{8}, 2^{8}, 1^{4}$ & & & \\
\hline & 3 & $3^{16}, 2^{2}$ & & & \\
\hline & & $5^{7}, 3^{2}, 2^{4}, 1^{3}$ & & & \\
\hline & others & $6^{2}, 5^{3}, 4^{2}, 3^{2}, 2^{4}, 1^{3}$ & & & \\
\hline$\left(B_{2}\right)^{(2)}$ & 2 & $4^{7}, 3^{7}, 1^{3}$ & & & \\
\hline \multirow[t]{3}{*}{$B_{2}$} & 2 & $4^{6}, 3^{4}, 2^{5}, 1^{6}$ & & & \\
\hline & & $5^{6}, 4^{4}, 1^{6}$ & & & \\
\hline & others & $7,5^{4}, 4^{4}, 3,1^{6}$ & & & \\
\hline$A_{2}+\tilde{A}_{1}$ & 2 & $4^{6}, 3^{4}, 2^{8}$ & & & \\
\hline & & $3^{16}, 2^{2}$ & & & \\
\hline & others & $5^{3}, 4^{2}, 3^{6}, 2^{4}, 1^{3}$ & & & \\
\hline$\tilde{A}_{2}$ & 2 & $4^{8}, 1^{20}$ & & & \\
\hline & & $3^{15}, 1^{7}$ & & & \\
\hline & others & $5^{7}, 3,1^{14}$ & & & \\
\hline$\left(A_{2}\right)^{(2)}$ & 2 & $4^{2}, 3^{12}, 1^{8}$ & & & \\
\hline$A_{2}$ & 2 & $4^{2}, 3^{12}, 1^{8}$ & & & \\
\hline & 3 & $3^{15}, 1^{7}$ & & & \\
\hline & others & $5,3^{13}, 1^{8}$ & & & \\
\hline$A_{1}+\tilde{A}_{1}$ & 2 & $2^{24}, 1^{4}$ & & & \\
\hline & & $3^{10}, 2^{8}, 1^{6}$ & & & \\
\hline & others & $4^{2}, 3^{6}, 2^{10}, 1^{6}$ & & & \\
\hline$\left(\tilde{A}_{1}\right)^{(2)}$ & 2 & $2^{21}, 1^{10}$ & & & \\
\hline$\tilde{A}_{1}$ & 2 & $2^{16}, 1^{20}$ & & & \\
\hline & others & $3^{7}, 2^{8}, 1^{15}$ & & & \\
\hline$A_{1}$ & 2 & $2^{16}, 1^{20}$ & & & \\
\hline & others & $3,2^{14}, 1^{21}$ & & & \\
\hline
\end{tabular}


Acknowledgements. We thank A. Thomas, A. Premet and D. Nakano for helpful discussions. We thank the referee for checking the results of this paper carefully. In particular, we are extremely grateful that a discrepancy was noted in characteristic 2 , which we eventually traced back to errors in the representatives of orbits $D_{r}\left(a_{i}\right)$ in [16] (see $\S 1.1$ ).

\title{
References
}

1. H. Azad, M. Barry and G. Seitz, 'On the structure of parabolic subgroups', Comm. Algebra 18 (1990) no. 2, 551-562; MR 1047327 (91d:20048).

2. M. Bate, B. Martin, G. Röhrle and R. Tange, 'Complete reducibility and separability', Trans. Amer. Math. Soc. 362 (2010) no. 8, 4283-4311.

3. N. Bourbaki, Éléments de mathématique. Groupes et algèbres de Lie. Chapitres 4, 5 et 6 (Masson, Paris, 1981); MR 0647314.

4. A. M. Cohen and B. N. Cooperstein, 'The 2-spaces of the standard E6 $(q)$-module', Geom. Dedicata 25 (1988) no. 1-3, 467-480; Geometries and groups (Noordwijkerhout, 1986), MR 925847 (89c:51013).

5. S. Herpel, 'On the smoothness of centralizers in reductive groups', Trans. Amer. Math. Soc. 365 (2013) no. 7, 3753-3774; MR 3042602.

6. S. Herpel and D. I. StewART, 'On the smoothness of normalisers, the subalgebra structure of modular Lie algebras and the cohomology of small representations', Doc. Math. 21 (2016) 1-37.

7. J. C. Jantzen, Representations of algebraic groups, 2nd edn, Mathematical Surveys and Monographs 107 (American Mathematical Society, Providence, RI, 2003); MR 2015057 (2004h:20061).

8. P. B. Kleidman, 'The maximal subgroups of the Chevalley groups $G_{2}(q)$ with $q$ odd, the Ree groups ${ }^{2} G_{2}(q)$, and their automorphism groups', J. Algebra 117 (1988) no. 1, 30-71; MR 955589 (89j:20055).

9. R. LAWther, 'Jordan block sizes of unipotent elements in exceptional algebraic groups', Comm. Algebra 23 (1995) no. 11, 4125-4156; MR 1351124 (96h:20084).

10. R. Lawther, Correction to: "Jordan block sizes of unipotent elements in exceptional algebraic groups" [Comm. Algebra 23 (1995) no. 11, 4125-4156; MR 1351124 (96h:20084)], Comm. Algebra 26(8) (1998) 2709; MR 1627924 (99f:20073).

11. S. P. Le Halleur, 'Subgroups of maximal rank of reductive groups', Autour des schémas en groupes, Panor. Synthèses (2014) 42-43.

12. M. W. LiEBECK and J. SAXL, 'On the orders of maximal subgroups of the finite exceptional groups of Lie type', Proc. Lond. Math. Soc. (3) 55 (1987) no. 2, 299-330; MR 896223 (89b:20068).

13. M. W. Liebeck and G. M. Seitz, Unipotent and nilpotent classes in simple algebraic groups and Lie algebras, Mathematical Surveys and Monographs 180 (American Mathematical Society, Providence, RI, 2012); MR 2883501.

14. N. Spaltenstein, 'Nilpotent classes in Lie algebras of type $F_{4}$ over fields of characteristic 2', J. Fac. Sci. Univ. Tokyo Sect. 1A 30 (1984) no. 3, 517-524; MR 731515.

15. T. A. Springer and R. Steinberg, 'Conjugacy classes', Seminar on algebraic groups and related finite groups (The Institute for Advanced Study, Princeton, N.J., 1968/69), Lecture Notes in Mathematics 131 (Springer, Berlin, 1970) 167-266; MR 0268192 (42 \#3091).

16. University of Georgia Vigre Algebra Group: D. J. Benson, P. Bergonio, B. D. Boe, L. Chastkofsky, B. Cooper, G. M. Guy, J. Hower, M. Hunziker, J. J. Hyun, J. Kujawa, G. Matthews, N. Mazza, D. K. Nakano, K. J. Platt and C. Wright, 'Varieties of nilpotent elements for simple Lie algebras. II. Bad primes', J. Algebra 292 (2005) no. 1, 65-99; MR 2166796 (2006k:14083).

\author{
David I. Stewart \\ School of Mathematics and Statistics \\ University of Newcastle \\ Herschel Building \\ Newcastle NE1 7RU \\ United Kingdom
}

dis20@cantab.net 